\title{
Protection of primary users in dynamically varying radio environment: practical solutions and challenges
}

\author{
Pawel Kryszkiewicz ${ }^{1}$, Hanna Bogucka ${ }^{1 *}$ and Alexander M Wyglinski ${ }^{2}$
}

\begin{abstract}
One of the primary objectives of deploying cognitive radio (CR) within a dynamic spectrum access (DSA) network is to ensure that the legacy rights of incumbent licensed (primary) transmissions are protected with respect to interference mitigation when unlicensed (secondary) communications are simultaneously operating within the same spectral vicinity. In this article, we present non-contiguous orthogonal frequency division multiplexing (NCOFDM) as a promising and practical approach for achieving spectrally agile wireless data transmission that is suitable for secondary users (SUs) to access fragmented spectral opportunities more efficiently. Furthermore, a review of the current state-of-the-art is conducted with respect to methods specifically designed to protect the transmissions of the primary users (PUs) from possible interference caused by nearby SU transceivers employing NC-OFDM. These methods focus on the suppression of out-of-band (OOB) emissions resulting from the use of NCOFDM transmission. To achieve the required OOB suppression, we present two practical approaches that can be employed in NC-OFDM, namely, the insertion of cancellation carriers and windowing. In addition to the theoretical development and proposed improvements of these approaches the computer simulation results of their performance are presented. Several real-world scenarios regarding the coexistence of both PU and SU signals are also studied using actual wireless experiments based on software-defined radio. These simulation and experimental results indicate that $O O B$ suppression can be achieved under real-world conditions, making NC-OFDM transmission a viable option for CR usage in DSA networks.
\end{abstract}

\section{Introduction}

The idea of cognitive radio (CR) encompasses opportunistic and dynamic access to spectrum resources that might be available at a certain location and time. These resources, called spectrum holes, especially in metropoli$\tan$ areas, can potentially be fragmented with several non-contiguous spectral bands of different width. Moreover, the availability of these spectrum holes may dynamically change over time, as the licensed users (primary users-PUs) enter into and depart from a given location. There has been a substantial amount of research conducted with respect to finding suitable technologies capable of aggregating the available spectrum adaptively according to dynamics of spectrum holes availability, and to support the transmissions of the secondary users

\footnotetext{
* Correspondence: hbogucka@et.put.poznan.pl

${ }^{1}$ Chair of Wireless Communications, Poznan University of Technology,

Poznan, Poland

Full list of author information is available at the end of the article
}

(SUs) in a spectrally efficient manner. In order to use the fragmented spectrum, an SU radio transceiver must be able to shape its emission to make best use of available resources while simultaneously respecting the incumbent spectral accessing rights of the PUs.

The key for achieving a spectrally agile waveform that enables the coexistence of both PU and SU transmissions within a specified spatial, temporal, and spectral vicinity is to exert strict control over the spectral extent of the transmitted signal. One spectrally agile waveform approach that has been receiving significant attention in recent years is noncontiguous orthogonal frequency division multiplexing (NC-OFDM) [1,2], which is based on the popular orthogonal frequency division multiplexing (OFDM) transmission technique. One of the primary advantages of using NC-OFDM within the context of a dynamic spectrum access (DSA) network is that it provides the flexibility of deactivating, or nulling, specific subcarriers with zeros as input values such that there is 
no SU transmit power at frequency locations corresponding to the presence of PU emissions.

Despite its advantages, NC-OFDM possesses several substantial technical issues that need to be resolved in order to make this form of wireless data transmission within a DSA environment a viable option. One of these issues is the shape of the NC-OFDM spectrum outside of the intended transmission bandwidth, which is known to be relatively high when left untreated due to the Sinc pulse shapes of the individual data-bearing subcarriers. Consequently, if PU transmissions are located next to a collection of data-bearing subcarriers belonging to an NC-OFDM signal, this may result in the former experiencing an unacceptable level of interference from the latter. Therefore, it is essential that the spectral shape of the NC-OFDM waveform is treated such that the out-of-band $(\mathrm{OOB})$ radiation is minimized.

In addition to the issue of OOB interference, OFDMbased waveforms are generally characterized by relatively high peak-to-average-power ratio (PAPR), which makes the transmit signal vulnerable to nonlinear distortions, such as signal clipping in high-power amplifiers. If signal clipping does occur, the resulting transmission spectrum will broaden, thus yielding a potential interference situation with adjacent PU signals. Consequently, it is important to investigate suitable methods for reducing the PAPR of NC-OFDM transceivers with the goal of mitigating OOB interference. There have been a number of articles dealing with this problem, suggesting either PAPR reduction methods (see an overview of these methods in [3] and the references therein), signal predistortion (e.g., [4]) or the linearization methods of a power amplifier. However, further development of these methods is required to make them sufficiently practical for the purposes of realizing transceiver implementations in actual real-world scenarios, such that the choice of an appropriate method should be able to handle the time-varying radio transmission environment, including dynamically changing types of the PU transmissions. Simultaneously, these methods should aim at achieving reasonable computational complexity, negligible performance degradation of the SU transmission, and low energy costs.

In this article, we present an investigation of spectrally agile waveforms based on NC-OFDM and assess their suitability for achieving SU transmissions that are capable of respecting the rights of incumbent PU signals. In Section 2, we present an overview of NC-OFDM transmission within the context of a cognitive radio-based DSA network. We then review in Section 3 existing methods for achieving flexible spectral waveforms using NC-OFDM while simultaneously mitigating the effects of OOB interference. Section 4 provides a closer look at a promising technique for mitigating $\mathrm{OOB}$ interference that combines the insertion of so-called cancel lation carriers (CCs) with OFDM symbol-based windowing. Moreover, we present an enhanced optimization algorithm with reduced computational complexity and reduced energy costs. Finally, the proposed OOB interference reduction approach for NC-OFDM is evaluated using actual wireless transceivers based on softwaredefined radio (SDR) technology within a controlled environment, and the results of these experiments are presented and discussed in Section 5.

\section{Spectrally agile multicarrier waveform framework}

A conventional wireless transmission system is usually allocated a specific frequency band for data communications. These wireless transmissions are usually licensed, which means they possess exclusive rights to the assigned frequency bands. Although much of the wireless spectrum up to $3 \mathrm{GHz}$ has been assigned to licensed wireless applications, several measurements campaigns have shown that a substantial portion of the licensed frequency bands are underutilized across the temporal, spectral, and spatial domains [5]. To continue providing sufficient spectral bandwidth for satisfying both current and future wireless access needs, both spectrum policy makers and communication technologists have proposed an innovative approach with respect to the wireless spectrum usage via opportunistic spectrum access (OSA). Relative to traditional approaches for accessing spectrum, OSA allows for unlicensed wireless users to temporarily "borrow" unoccupied licensed frequency bands [6]. However, these unlicensed (i.e., secondary) devices must still guarantee interference-free wireless access to incumbent licensed (i.e., primary) signals. In particular, it is essential that the OOB radiation generated by the $\mathrm{SU}$ wireless device is mitigated in order to prevent interference with PU wireless signals located in the frequency vicinity. Consequently, given this constraint on SU wireless transceivers, communication systems performing OSA require a level of spectral agility in order to operate in the presence of PU signals, especially when it comes to mitigating interference resulting from OOB radiation, as well as simultaneously transmitting across several unoccupied frequency bands that are fragmented across the wireless spectrum whose aggregate bandwidth satisfies the secondary transmission requirements.

Multicarrier modulation (MCM) possesses sufficient spectral agility in order to facilitate the transmission of data from unlicensed SU transmitters across several fragmented frequency bands simultaneously even in the presence of licensed PU signals, thus resulting in an increase in spectrum utilization [7]. In particular, subcarriers located in the frequency vicinity of unoccupied 
wireless spectrum can be used for transmitting data while those sub-carriers that could potentially interfere with nearby PU signals can be deactivated or nulled. However, simply deactivating subcarriers for the purposes of OOB interference mitigation may not be sufficient for the neighboring PUs' interference tolerance levels. Moreover, in addition to achieving a required level of OOB interference within a given spectrum mask, an SU transmitter performing OSA must be capable of tailoring its spectral characteristics dynamically in order to avoid interference with the dynamically changing incumbent licensed PU transmissions. Finally, most MCM transmission approaches possess the possibility of exhibiting large envelope variations in the time domain that is often characterized by a high PAPR. This results from the combination of the subcarrier signals into a single composite multicarrier waveform in the time domain. When high PAPR occurs, the resulting transmission spectrum broadens and produces $O O B$ interference regardless of whether the initial spectral waveform has been properly shaped at the transmitter for low OOB interference.

Overall, non-contiguous MCM techniques have been recognized as a suitable candidate for OSA due to their potential for achieving spectrally efficient communications by exploiting fragmented unoccupied spectrum while simultaneously achieving high data rates $[8,9]$. In fact, this form of data transmission approach is well-suited for future wireless communication systems, including CR systems [10]. As mentioned before, the NCOFDM scheme possesses the ability to efficiently use fragmented spectrum opportunities as well as perform spectrum shaping in order to suppress interference that may affect nearby primary wireless transmissions. To counteract the potential for significant $\mathrm{OOB}$ interference resulting from NC-OFDM transmission, which can negatively affect neighboring wireless signals, several techniques have been proposed in the literature that are designed to significantly suppress these sidelobes in order to make coexistence between PUs and SUs feasible. On the other hand, the OOB reduction process can potentially increase the computational complexity and energy (power) utilization. Given the possible constraints of limited computational and energy resources available via a user equipment, a practical approach to this problem is needed that achieves a balance between the OOB interference mitigation efficiency and its associated costs.

\section{OOB reduction techniques for spectrally agile multicarrier waveforms}

The dilemma of how to mitigate the OOB interference in multicarrier systems has attracted substantial interest over the last decade. In this section, we present an overview of the major achievements in this field, and indicate two methods that are particularly attractive for the application in CR framework.

\subsection{State-of-art techniques for OOB radiation reduction}

The simplest method for achieving OOB interference reduction is to reserve a number of edge (guard) subcarriers (GS) to serve as a spectral buffer between PU and SU transmissions [7], i.e., deactivation of subcarriers. Although simple to implement, this method significantly decreases the spectral efficiency and does not provide sufficient $\mathrm{OOB}$ interference reduction in most scenarios.

Another approach to the OOB power reduction of an OFDM signal is to spectrally shape each individual subcarrier spectrum [7]. We will discuss this simple method called windowing $(\mathrm{W})$ in the following subsection in greater detail. In the adaptive symbol transition (AST) method [11], similar to W, the time-domain samples in the transition region between consecutive symbols are chosen adaptively in order to minimize the OOB power. For the AST algorithm, the information about symbols mapped to each subcarrier is needed in order to assess the amount of $\mathrm{OOB}$ interference in the neighboring frequency bands. A mean-square-error (MSE) minimization method is used to determine the values of the time-domain samples in the transition region. The primary drawbacks of this method are high computational complexity and reduced throughput.

Another method, called constellation expansion (CE) [12], adjusts the modulated data symbols transmitted per subcarrier such that the OOB interference can be reduced while simultaneously not losing any data information or causing distortion. This is achieved by enlarging the modulation constellation and by allowing data symbols to be represented by any one of the two constellation points. As a result, the minimum distance between the constellation points is reduced, and the biterror-rate (BER) performance decreases.

Another method, called subcarriers weighting (SW) $[13,14]$, minimizes the signal OOB interference level by multiplying the data subcarriers by optimized real weighting coefficients. At the receiver, data symbols transmitted using the weighted subcarriers can be viewed as distorted, particularly for the high values of the weighting coefficients. Consequently, the authors suggest to impose a constraint on the weighting coefficients values. Simulation results exhibit significant OOB interference suppression. Some modifications to this method have been made in [15], where maximization of the channel capacity combined with OOB interference mitigation is addressed.

In the multiple-choice sequences (MCS) [16] method, for each sequence of data symbols to be transmitted in an OFDM symbol, a set of corresponding sequences 
representing it is calculated. The sequence yielding the lowest interference to adjacent bands is then chosen from this set and transmitted. To retrieve the initial data sequence at the receiver the identification number of the selected sequence has to be provided, what requires additional control channel for this side-information. A variant of the MCS method with reduced computational complexity is presented in [17]. In this method, the corresponding sequences are generated through the data symbols phases rotation of the multiple of $\pi / 2$, and thus a limited number of possible sets of sequences must be examined to choose the optimum one. As the OFDM edge subcarriers possess the strongest influence on the OOB radiation, only those subcarriers are altered. Another variant of the MCS method involves its merging with other spectrum shaping algorithms, e.g., in [18] the authors combined the MCS method with both SW and CCs method.

Polynomial cancellation coding (PCC) has been proposed in [19] and revisited in [20]. This method not only reduces the OOB radiation but also lowers the OFDM signal sensitivity to phase and frequency errors. As neighboring subcarriers have firmly aligned spectra, the adjacent subcarriers are modulated with the same, appropriately scaled data symbol in order to reduce the sidelobes power. This is usually done for groups of two or three subcarriers. Although this method reduces the system throughput, this effect can be weakened as the cyclic prefix $(\mathrm{CP})$ does not have to be added and coded redundancy can be used to increase SNR.

Another method for achieving OOB interference reduction, called spectral precoding (SP), has been described in $[21,22]$. In this method, the correlation between the data-symbols transmitted on subcarriers is introduced by block-coding. The code-generating matrix is chosen so as to minimize the OOB radiation power. The SP method provides the lowest OOB interference levels relative to other methods simulated in [21]. On the other hand, it has been observed that the OOB interference suppression is not so high when the $\mathrm{CP}$ is applied.

Another method for reducing $\mathrm{OOB}$ interference, called extended active interference cancellation (EAIC) [23], is based on the insertion of special carriers that are designed to negatively combine with high-power sidelobes caused by the data subcarriers. The AIC subcarriers can be placed inside the adjacent transmission spectrum, usually at frequency locations that are nonorthogonal to the SU data subcarriers. The main drawback of this method results from this lack of orthogonality and thus, data symbols distortion. A variant of the EAIC method was presented in [24], where the sidelobe suppression approach was improved by using a long time-domain cancellation signal spanning over a number of consecutive OFDM symbols. This method results in an increase of BER due to increased interference relative to the method presented in [23]. In [25], this method is improved by introducing the constraint on the selfinterference power level.

An interesting approach to the mitigation of $\mathrm{OOB}$ interference, called partial response signaling (PRS) [26], makes the values on each subcarrier dependent on the subsequent OFDM symbols. This can be done by independent lowpass filters on each input of the inverse fast Fourier transform (IFFT) block. Although relatively substantial OOB interference suppression can be achieved even with very low order (2-3) filters, the reception of such a signal requires either a slicer or a Viterbi detector when treating PRS filtering after being influenced by the multipath propagation channel.

An observation that the $\mathrm{OOB}$ radiation is the result of the time domain non-continuity between subsequent OFDM symbols was the basis for a spectrum shaping method presented in [27]. This method is called N-continuous OFDM (NC). The continuity of 0 th to $\mathrm{Nth}$ order derivatives at the ends of the OFDM symbols is achieved by adding low power, complex-valued quantities to each active data subcarrier at the input of a IFFT block.

An entire class of methods that support the protection of PU signals from the effects of OOB interference is based on the use of power allocation schemes that not only maximize the throughput but also reduce the OOB interference power, e.g., refer to methods presented in $[2,28,29]$. However, as these approaches might be seen as part of radio resources management they will not be investigated here further.

Finally, the concept of modulated filterbanks (MFB) can be also successfully applied to suppress the sidelobes of the OFDM transmission [30]. MFB can be used for sidelobe suppression by applying them over the OFDM spectrum such that the series of bandpass filters allows only the required spectrum to pass through it while rejecting the unwanted OOB radiations in every subband.

\subsection{Windowing}

Windowing (W) is usually applied to the OFDM symbol time-domain samples with CP. The use of windowing is shown in Figure 1. The time-domain OFDM symbol of duration $N+N_{\mathrm{CP}}$ samples, where $N_{\mathrm{CP}}$ is the duration in samples of the CP, is extended cyclically with $\beta$ samples at the end of the considered symbol. This extension is referred to as the cyclic suffix (CS). If we denote the time-domain signal for a single OFDM symbol as a vector $\mathbf{x}=\left\{x_{-\beta-N_{\mathrm{CP}}}, \ldots, x_{N-1+\beta}\right\}$, the OFDM-symbol timedomain samples are defined by vector $\boldsymbol{y}=\left\{y_{k}\right\}$, which 


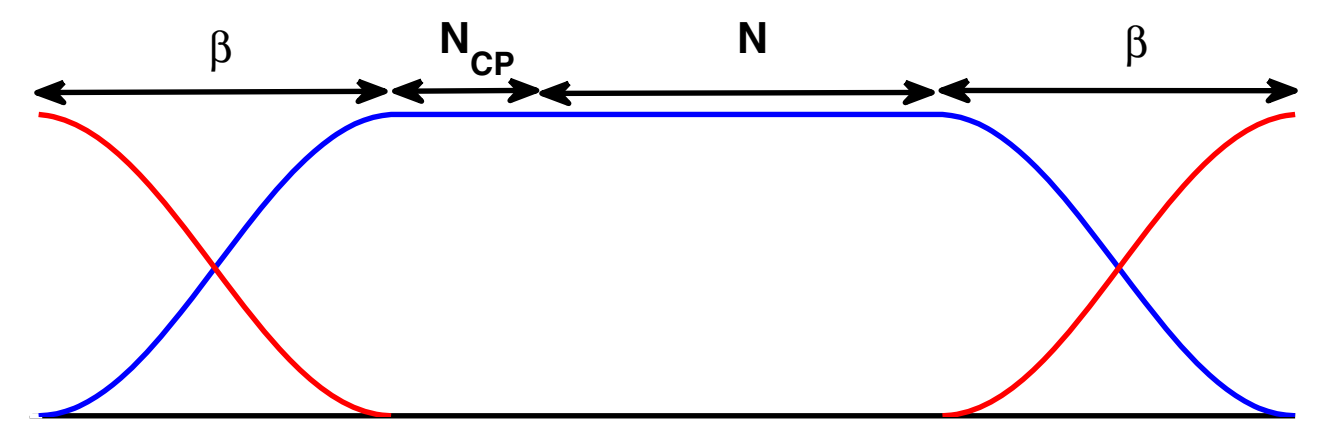

Figure 1 Windowed OFDM symbols; red lines denote the preceding and the following OFDM symbol

results from the multiplication of the vector $\mathbf{x}=\left\{x_{k}\right\}$ by the window shape $\mathbf{w}=\left\{w_{k}\right\}$, namely:

$$
y_{k}=w_{k} x_{k}
$$

where $k=-\beta-N_{\mathrm{CP}}, \ldots, N-1+\beta$. In [31], it has been shown that the largest sidelobe suppression is achieved when the Hanning window is applied. In case of the Hanning window, $\mathbf{w}=\left\{w_{k}\right\}$ possesses the following form:

$$
w_{k}=\left\{\begin{array}{ll}
0.5+0.5 \cos \left(\pi \frac{k+N_{\mathrm{CP}}}{\beta}\right), & k \in\left\{-\beta-N_{\mathrm{CP}}, \ldots,-1-N_{\mathrm{CP}}\right\} \\
1, & k \in\left\{-N_{\mathrm{CP}}, \ldots, N-1\right\} \\
0.5+0.5 \cos \left(\pi \frac{k-N}{\beta}\right), & k \in\{N, \ldots, N-1+\beta\}
\end{array} .\right.
$$

Referring to Figure 1, it is worth mentioning that to provide a relatively small throughput decrease, consecutive symbols overlapping with each other by $\beta$ samples can yield an effective OFDM symbol duration of $N+$ $N_{\mathrm{CP}}+\beta$ samples.

The primary advantages of this method is its low computational complexity, independence of the modulated data, and its suitability for NC-OFDM. When employed by a CR communication system attempting to access the available spectrum in a dynamically varying radio environment, it is also important that the length and shape of the applied window can be also altered dynamically. This method is the most suitable for minimizing the interference in the PU transmission that is relatively distant in frequency from SU transmission band $[7,31]$. The main drawback of this method is the decrease of throughput caused by the addition of the CS.

\subsection{Cancellation carriers}

The CCs method [32] takes advantage of the spectrum shape of each subcarrier in order to reduce the resulting OOB interference level. As each OFDM symbol possesses a limited time duration, this can be interpreted as cutting out a part of an infinitely long OFDM symbol by a rectangular window. In other words, the spectrum of each subcarrier is convolved with a sinc function, thus widening the spectral overlapping regions with the other subcarrier spectra. Although this is generally the primary reason for the existence of high OOB interference, this phenomenon can be manipulated in a positive fashion using the CCs method, where a subset of active subcarriers are selected for the sole purpose of cancelling the OOB interference of the adjacent subcarriers. As the subcarriers closest to the spectrum edge have the strongest influence on the $\mathrm{OOB}$ radiation, they are usually chosen to carry the cancelling signal, and they do not support any data transmissions themselves. The sidelobes of these subcarriers are intended to negatively combine with the sum of the sidelobes resulting from the active data-bearing subcarriers, thus potentially reducing the overall OOB interference levels as shown in Figure 2.

The values of the cancellation subcarriers have to be calculated for each OFDM symbol separately since the independent modulated data symbols cause different OOB interference levels. Thus, several frequency-sampling points are defined in order to determine the values of the OOB signal spectrum at the corresponding frequencies. These frequency-sampling points describe the optimization region in which the estimates of the spectrum values resulting from the spectral superposition of the data subcarriers (DCs) and the CCs have to be calculated. The optimization problem to be solved for each OFDM symbol can be defined as follows:

$$
\min _{\mathbf{s}_{\mathbf{c}}}\left\|\mathbf{P}_{\mathrm{CC}}^{(\delta \times \gamma)} \mathbf{s}_{\mathbf{c}}+\mathbf{P}_{\mathrm{DC}}^{(\delta \times \alpha)} \mathbf{s}_{\mathbf{d}}\right\|^{2}
$$

where $\mathbf{P}_{\mathrm{CC}}^{(\delta \times \gamma)}$ is the matrix of dimensions $(\delta \times \gamma)$ transforming the vector of the CCs values $\mathbf{s}_{\mathbf{c}}$ of length 7 to the spectrum estimates. For the DCs, the matrix 


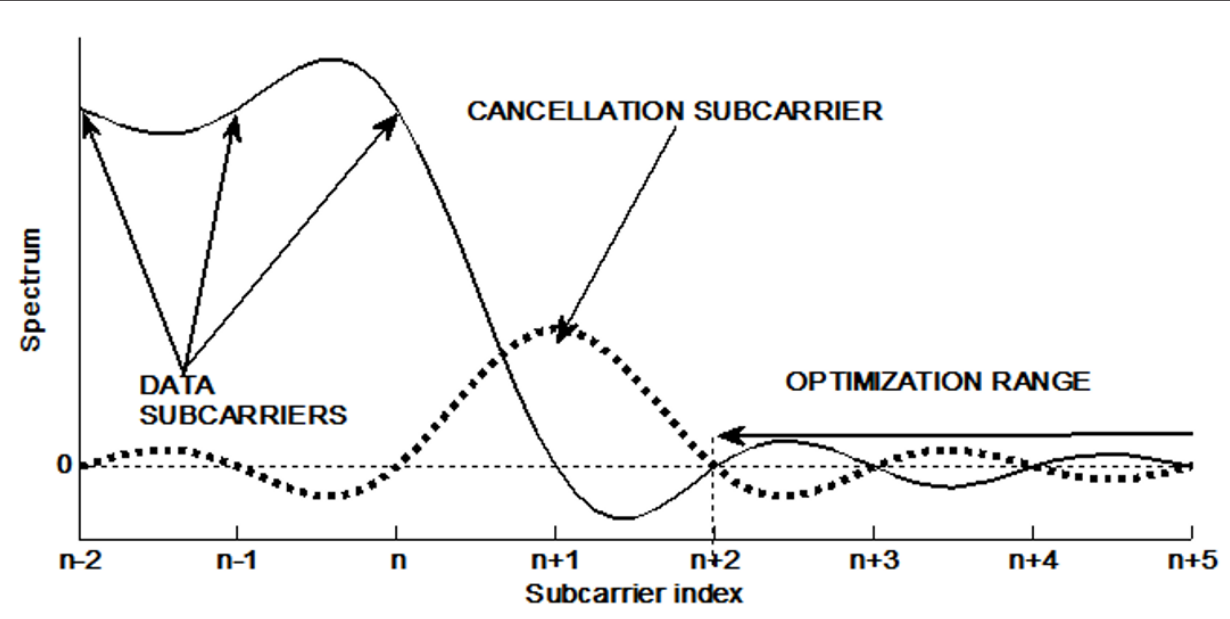

Figure 2 The diagram of spectrum resulting from superposition of data sub-carriers spectrum (solid line) and the cancellation subcarrier spectrum (dotted line). Optimization region marked, no CP used.

of dimensions $(\delta \times \alpha)$ and vector $\mathbf{s}_{\mathbf{d}}$ of length $a$ perform the same role. However, as the authors of this method have found, such an optimization approach may result in a higher power level for the CCs relative to the DCs. Consequently, the additional constraint has been introduced to limit this effect:

$$
\left\|\mathbf{s}_{\mathbf{c}}\right\|^{2} \leq \Pi_{\mathrm{CC}}
$$

where $\Pi_{\mathrm{CC}}$ is the maximum allowable power for CCs. Although the solution of (3) is widely known, and has been presented in $[33,34]$, the constraint (4) increases the computational complexity of the optimization problem significantly, requiring us to solve the Lagrange inequality for each OFDM symbol, which might become infeasible for wideband transmissions possessing a large number of subcarriers.

Another drawback of the $\mathrm{CC}$ method, apart from the computational complexity, is the link-performance deterioration, i.e., an increase of the BER. This is due to the fact that an OFDM system usually operates under the total power constraint. If part of an OFDM symbol energy is sacrificed to the CCs, the remaining energy that can be used for data transmission is reduced, and this naturally results in an SNR loss and corresponding BER degradation.

Nevertheless, the $\mathrm{CC}$ algorithm has been extensively investigated, and a number of modifications and combinations of the $\mathrm{CC}$ algorithm with other methods has been presented in the literature, e.g., refer to proposed approaches in [35-37]. For example, active interference cancellation (AIC) [33] is a method similar to CCs solution, where in addition to the OFDM edge-subcarriers several other subcarriers inside the PU transmission band are also used to minimize the OOB radiation.
However, as shown in the aforementioned paper, the AIC subcarriers inside the PU transmission band possess a negligible influence on the OOB interference. Moreover, they can significantly increase the computational complexity of the resulting implementation. The CCs method is very flexible in terms of defining the number of cancellation subcarriers and their power levels. Moreover, some of its shortcomings can be efficiently equalized if the W method with a parameterdefined window duration is also applied.

In Table 1, the main properties of the OOB power reduction methods discussed in this section are summarized. From this summary, we can conclude that the methods best-suited for the application in CR and the DSA networks are the CC and W methods. Moreover, the combination of these two methods have the potential for some promising performance improvements in terms of flexibility. In the next section, we discuss the combination of these methods in detail, and propose new algorithm that allows for reduction of its computational complexity, reduction of the power assigned to the CCs (considered wasted for the transmission of symbols bearing no information), improvement of BER and thus, for the implementation of this algorithm in practical systems.

\section{Advances of the state-of-the-art in the OOB power reduction: promising combination of windowing and CCs technique}

4.1 Reduced-complexity reduced-power combined CCs and windowing

The combination of CCs with windowing seems to be a promising spectrum shaping mechanism. While windowing method provides better $\mathrm{OOB}$ interference 
Table 1 Summary of the main features of the OOB reduction methods for NC-OFDM

\begin{tabular}{|c|c|c|c|c|c|c|c|c|c|c|c|c|c|}
\hline Properties & GS & W & AST & CE & $\mathrm{CCs}^{*}$ & SW & MCS & PCC & SP & EAIC & PRS & NC & MFB \\
\hline Increase of PAPR & No & No & No & No & Yes & No & No & No & No & Yes & No & No & Yes \\
\hline Computational complexity & Low & Low & High & High & High & High & High & Low & High & High & High & High & High \\
\hline Side information control channel & No & No & No & No & No & No & Yes & No & No & No & No & No & No \\
\hline Suitability for high order modulations & Yes & Yes & Yes & No & Yes & No & No & Yes & Yes & No & No & Yes & Yes \\
\hline Seemless reception & Yes & No & No & No & Yes & Yes & No & No & No & Yes & No & No & Yes \\
\hline Decrease of throughput & High & High & Low & No & Low & No & High & High & Low & Low & No & No & No \\
\hline Increase of BER & No & No & No & Yes & Yes & Yes & No & No & No & No & Yes & Yes & No \\
\hline
\end{tabular}

Guard Subcarriers (GS), Windowning (W), Adaptive Symbol Transition (AST), Constellation Expansion (CE), Cancellation Carriers - basic method, without modifications (CCs*), Subcarriers Weighting (SW), Multiple-Choice Sequences (MCS), PCC, Spectral Precoding (SP), Extended Active Interference Cancellation (EAIC), Partial Response Signaling (PRS), N-continuous OFDM (NC), Modulated Filter Banks (MFB)

mitigation for spectrum components more distant from occupied OFDM band on the frequency axis, the CCs method has the same behavior for components closer to the OFDM nominal band as shown in [34]. Thus, the combination of both methods, which was also presented in [34], provides additional degrees of freedom as the number of CCs and window shapes can be altered to fulfill the transmission requirements. In this section, we present several additional enhancements to this combined approach, thus yielding a reduction in the computational complexity, reduction of the energy-loss (energy inefficiency) due to the use of the CCs, and an improvement of the BER performance.

The system that we consider in this research consists of a conventional OFDM modulator, where the CCs unit, which performs the CCs algorithm, is employed prior to the $N$-size IFFT block, and windowing is applied to the time domain signal after extending it with the CP. The resulting OFDM-modulated signal after the OOB interference reduction process is then fed to the digital-to-analog converter and the IF/RF (Intermediate Frequency/Radio Frequency) front-end.

The CCs optimization formula must be designed for the time domain windowed signal y. Suppose we denote the input of the IFFT as the vector $\mathrm{s}=\left\{s_{-N / 2}, \ldots, s_{N / 2-1}\right\}$, which contains zeros except for the elements indexed as $\mathrm{c}=\left\{c_{1}, \ldots, c_{\gamma}\right\}$ and $\mathbf{d}=\left\{d_{1}, \ldots, d_{\alpha}\right\}$, where the CCs symbol values and the data symbols are inserted, respectively. The optimization of cancellation symbols is based on the estimation of the spectrum values resulting from the superposition of the spectra of each CC and DC. The time-domain OFDM symbol vector $\mathbf{y}$ elements can be mathematically expressed as:

$$
y_{k}=w_{k} \sum_{n=-N / 2}^{N / 2-1} s_{n} \exp \left(j 2 \pi \frac{n k}{N}\right),
$$

where this OFDM-symbol spectrum estimate at frequency bin $l$ can be derived by preforming the $M$-times upsampled FFT for this point $b_{l}$ using the following approach:

$$
\begin{aligned}
b_{l} & =\sum_{k=-N_{\mathrm{CP}}-\beta}^{N+\beta-1} w_{k} \sum_{n=-N / 2}^{N / 2-1} s_{n} \exp \left(j 2 \pi \frac{n k}{N}\right) \exp \left(-j 2 \pi \frac{l k}{N M}\right) \\
& =\sum_{n=-N / 2}^{N / 2-1} s_{n} \sum_{k=-N_{\mathrm{CP}}-\beta}^{N+\beta-1} w_{k} \exp \left[j 2 \pi \frac{k}{N}\left(n-\frac{l}{M}\right)\right] \\
& =\sum_{n=-N / 2}^{N / 2-1} s_{n} p_{n, l} .
\end{aligned}
$$

For a set of frequency-sampling points $\mathbf{l}=\left\{l_{1}, \ldots, l_{\delta}\right\}$ defined in the optimization region, and for $n \in \mathbf{c}$, the coefficients $p_{n, l}$ are the elements of the matrix $\mathbf{P}_{\mathrm{CC}}^{(\delta \times \gamma)}$, and can be pre-calculated. Similarly, for the data carriers, when $n \in \mathbf{d}, p_{n, l}$ defines the matrix $\mathbf{P}_{\mathrm{DC}}^{(\delta \times \alpha)}$, and can be calculated off-line.

The commonly used optimization problem definition can be expressed using the Equation (3). Recall that the aim of this research is to minimize the OOB interference level, which implies solving the following optimization framework:

$$
\min _{\mathbf{s}_{\mathbf{c}}}\left\|\mathbf{P}_{\mathrm{CC}}^{(\delta \times \gamma)} \mathbf{s}_{\mathbf{c}}+\mathbf{P}_{\mathrm{DC}}^{(\delta \times \alpha)} \mathbf{s}_{\mathbf{d}}\right\|^{2}
$$

where $\mathbf{s}_{\mathbf{d}}$ and $\mathbf{s}_{\mathbf{c}}$ contain complex values modulating data carriers and CCs respectively, and form the subvectors of vector s created by the $\mathbf{d}$ and $\mathbf{c}$ indexed cells, respectively. The solution of this problem yields the values of CCs $\mathbf{s}_{\mathbf{c}}$, namely:

$$
\mathbf{s}_{\mathbf{c}}=-\mathbf{P}_{\mathrm{CC}}^{(\delta \times \gamma)}+\mathbf{P}_{\mathrm{DC}}^{(\delta \times \alpha)} \mathbf{s}_{\mathbf{d}},
$$

where []$^{+}$denotes the pseudoinverse. Although such a solution is relatively fast with respect to computational complexity, since the multiplication of vector sd by a precalculated matrix is performed for each OFDM symbol, it suffers from several issues. 
First, as shown in [32], several parts of the OFDM symbol power will need to be allocated to the CCs. Thus, in practical systems with an appropriately chosen power constraint, the SNR for the data carriers is reduced by the estimated value:

$$
\xi=10 \log _{10}\left(\frac{\left\|\mathbf{s}_{\mathbf{c}}\right\|^{2}+\left\|\mathbf{s}_{\mathbf{d}}\right\|^{2}}{\left\|\mathbf{s}_{\mathbf{d}}\right\|^{2}}\right) .
$$

The reference system in this case is the one that employs the nulled guard sub carriers on the subcarriers used by the CCs method in the proposed system. Another significant drawback is a substantial increase of the PAPR that is caused by the high power values transmitted on the CCs correlated with the DCs. Apart from the PAPR value, usually the probability of peaks occurrence is also taken into account since it is conceivable that the time-domain peaks possessing moderate instantaneous power can cause nonlinear distortions and performance deterioration that can prove to be much worse than the high power (strong) peaks occurring relatively infrequently. On the basis of this observation, the PAPR is measured with a certain probability $p$ PSPR. We will determine this metric later in this section when providing simulation results for a probability of $p$ PAPR $=10^{-3}$.

Finally, an important phenomenon when applying the $\mathrm{CC}$ method for OOB interference reduction is the occurrence of frequency-domain power peaks for frequencies assigned to the CCs. This is due to the fact that the CCs have to compensate for a number of DC sidelobes. A large power increase at the edges of the NC-OFDM frequency spectrum (where CCs are located) may be unacceptable according to the existing regulations that impose constraints on the transmission spectral masks. In order to provide some metric reflecting this problem, let us define the spectrum overshooting ratio (SOR) for a given probability psor of exceeding level $\varrho$ of the spectrum mask by the CCs power:

$$
\mathrm{SOR}=10 \log _{10}\left(\frac{\arg _{\varrho}\left[\operatorname{Pr}\left(S\left(f_{\mathrm{CC}}\right)>\varrho\right)=p_{\mathrm{SOR}}\right]}{\frac{1}{B_{\mathrm{SU}-\mathrm{CC}}} \int_{\mathrm{BU}_{\mathrm{SU}} \mathrm{CC}} S(f) d f}\right),
$$

where $S(f)$ is the power spectral density (PSD) function of the considered NC-OFDM secondary-user signal, $B_{\mathrm{SU}-\mathrm{CC}}$ is the ba ndwidth of the considered NC-OFDM secondary-user transmission used by the data subcarriers (excluding cancellation subcarriers frequency bands), and $f_{\mathrm{CC}}$ is any one of the frequencies belonging to CCs bands. This definition for the SOR can be interpreted as the logarithm of the PSD peaks of CCs with respect to the mean power level in data carriers band. The occurrence of these peaks is measured with probability $p_{\mathrm{SOR}}$. Note that in simulation results presented in the next subsection, $p_{\mathrm{SOR}}=10^{-1}$ will be considered. This probabilistic approach is required to take a varying characteristic of the PSD estimate into account.

To overcome the aforementioned problem with respect to an unacceptable power increase, we propose to supplement the optimization problem described by (7) with an additional, indirect constraint whose aim is to minimize the CCs power. The optimization problem is now defined as follows:

$$
\min _{\mathbf{s}_{\mathbf{c}}}\left\{\left\|\mathbf{P}_{\mathrm{CC}}^{(\delta \times \gamma)} \mathbf{s}_{\mathbf{c}}+\mathbf{P}_{\mathrm{DC}}^{(\delta \times \alpha)} \mathbf{s}_{\mathbf{d}}\right\|^{2}+\mu\left\|\mid \mathbf{s}_{\mathbf{c}}\right\|^{2}\right\},
$$

where $\mu$ factor is used to balance between the CCs power and resulting OOB power reduction. The solution of this problem can be derived by merging both conditions and related matrix operations, and results in the following vector of CCs, namely:

$$
\mathbf{s}_{\mathbf{c}}=-\left(\begin{array}{c}
\mathbf{P}_{\mathrm{CC}}^{(\delta \times \gamma)} \\
\sqrt{\mu} \mathbf{I}^{(\gamma \times \gamma)}
\end{array}\right)^{+}\left(\begin{array}{c}
\mathbf{P}_{\mathrm{CC}}^{(\delta \times \alpha)} \\
0
\end{array}\right) \mathbf{s}_{\mathbf{d}}=\mathbf{W} \mathbf{s}_{\mathbf{d}},
$$

where $\mathbf{I}^{(\gamma \times \gamma)}$ is a $\gamma$-size identity matrix, and $\mathbf{W}$ results from multiplication of the first two matrices in the above equation. Such an optimization has similar computational complexity to the optimization problem of (7), as only once for a given spectrum mask, and after the number of DCs and CCs are determined, the optimization (calculation of matrix $\mathbf{W}$ ) is implemented. Then, for each OFDM symbol, matrix-by-vector multiplication is carried out with pre-calculated matrix $\mathbf{W}$ elements. The performance and influence on various system parameters will be evaluated in the next section.

The optimization procedure described above significantly reduces the SNR loss typically found for a CCs method. This is obtained as a result of imposing a constraint on the value of the SOR, which consequently reduces the power assigned to CCs and increases power reserved for the DCs. Nevertheless, the reduced power available for data subcarriers still cause some deterioration of the reception quality. Therefore, we propose the following reception technique that makes use of the CCs inherent redundancy.

As the CCs are correlated with data symbols, these additional subcarriers can be used in the signal reception that might not only regain the power devoted to these subcarriers in the first place, but also make use of the frequency diversity for achieving a higher degree of robustness with respect to the frequency-selective fading. Let us consider Equation (12) as a process of generating redundancy symbols $\mathbf{s}_{\mathrm{c}}$ transmitted in parallel to data symbols $\mathbf{s}_{\mathrm{d}}$. This operation is conducted on the complex symbols, thus allowing us to employ the theory 
of complex-field block codes [38] for this problem formulation. To do so, let us rewrite Equation (12) in order to determine the systematic code generation matrix $\mathbf{G}$ of size $(\gamma+\alpha \times \alpha)$, namely:

$$
\mathbf{G}=\left(\begin{array}{c}
\mathbf{I}^{\alpha \times \alpha} \\
\mathbf{W}
\end{array}\right) \text {. }
$$

By changing the row order of presented matrix, the order of data and cancellation symbols can be kept, but for simplicity we will skip this operation. A simple reception mechanism designed for such codes is based on the zero-forcing criterion, for which the reception matrix is defined as:

$$
\mathbf{R}=(\mathbf{H G})^{+},
$$

where $\mathbf{H}$ is $(\gamma+\alpha \times \gamma+\alpha)$ diagonal matrix with channel coefficients for each of used subcarriers on its diagonal. This matrix should be used in the receiver after the FFT processing instead of an equalizer used in standard reception chain. The estimate of the data symbols $\hat{\mathbf{s}}_{\mathbf{d}}$ is achieved by the following operation:

$$
\hat{\mathbf{s}}_{\mathbf{d}}=\mathbf{R} \hat{\mathbf{s}}_{\mathbf{d}+\mathbf{c}}
$$

where $\tilde{\mathbf{s}}_{\mathbf{d}+\mathbf{c}}$ is a received vertical vector at the output of FFT block containing distorted and noisy values of data and cancellation subcarriers. Although the calculation of matrix $\mathbf{R}$ can be quite complex, it needs to be performed only once for each channel instance and subcarrier pattern. Moreover, with a systematic code implementation, this method may be treated as optional, reserved only for high performance, high quality reception.

Finally, let us derive a metric that indicates the potential throughput loss caused by introduction of CCs, windowing or the combination of CCs and W. This throughput loss can be assessed in comparison to a system not employing any OOB interference reduction method, in which all subcarriers are occupied by the DCs. Note that the actual system throughput depends not only on the number of data subcarriers but also on the power assigned to these subcarriers and the channel characteristic observed. Therefore, this metric indicates only potential throughput loss that results from the information signal bandwidth reduction due to introduction of the CCs and window duration extension, thus assuming the same transmit power and channel quality at each subcarrier. It is described by the following expression:

$$
R_{\text {loss }}=\left(1-\frac{1-\frac{\gamma}{\gamma+\alpha}}{1+\frac{\beta}{N+N_{\mathrm{CP}}}}\right) .100 \% .
$$

Note that the reference system for this definition, i.e., all subcarriers employed for data transmission, is prohibited from operating in the considered scenario, where the PU transmission protection is required and the $\mathrm{SU}$ sidelobes have to be reduced.

\subsubsection{Simulation results}

Below, we present the Monte Carlo simulation results using MATLAB and showing that our introduced modifications of the combined $\mathrm{CC}$ and $\mathrm{W}$ method improves the overall performance of the NC-OFDM system in several ways. In our experiments, we assumed $N=256$ subcarriers, where the subcarriers possessing the indices $\mathbf{d}=\{-100, \ldots,-62\} \cup\{-41, \ldots,-11\} \cup\{10, \ldots, 40\} \cup\{61, \ldots$, $101\}$ are occupied by the QPSK data symbols, and there are three CCs placed on each side of data carriers blocks, i.e. $\mathrm{c}=\{-103,-102,-101\} \cup\{-10,-9,-8\} \cup\{7,8$, $9\} \cup\{41,42,43\} \cup\{58,59,60\} \cup\{102,103,104\}$. The subcarriers pattern of four data subcarrier blocks is separated with narrowband PUs, e.g., program making and special events (PMSE) devices such as professional wireless microphones with bandwidth of $200 \mathrm{kHz}$. Note that an explanation of the wideband and narrowband PU signals and scenarios under consideration with respect to the coexistence of the PU and SU transmissions are given in the next section, with the real-world experimental results. The duration of the CP equals $N_{\mathrm{CP}}=16 \mathrm{sam}$ ples, but the $\beta=16$ samples of the Hanning window extension (equal to CS) are also used on each side of an OFDM symbol. The number of CCs and shaping window duration was chosen in such a way that the mean OOB interference power level is achieved at least $40 \mathrm{~dB}$ below the mean in-band power level for reasonable value of $\mu$, i.e., $\mu=0.01$. This OOB power attenuation is sufficient in order to respect several regulatory spectrum masks, e.g., IEEE802.11 g [39] or LTE user-equipment [40] Spectrum Emission Mask (SEM).

First, in Figure 3, we show the results of the OOB power reduction obtained for the following three methods under consideration: $\mathrm{CC}$ method, windowing, and combined CC and W scheme. The comparison has been performed for the schemes that present the same potential throughput loss metric, which for our evaluation system equals $R_{\text {loss }}=19.2 \%$. Such a potential throughput loss is obtained either from the CC method with $\gamma_{\mathrm{e}}=4$ CCs per edge of the DCs band, from the W method with Hanning window extension of $\beta=65$ samples, or from the combined CCs and W method with $\gamma_{\mathrm{e}}=3$ and $\beta=16$, i.e. the scenario described above. The PSDs were obtained for the signal before HPA using Welch's method after transmitting 10,000 random OFDM symbols. The spectrum was estimated in $4 N$ frequency sampling points using $3 \mathrm{~N}$-length Hanning windows. Note, that the windowing method achieves a high $\mathrm{OOB}$ power 


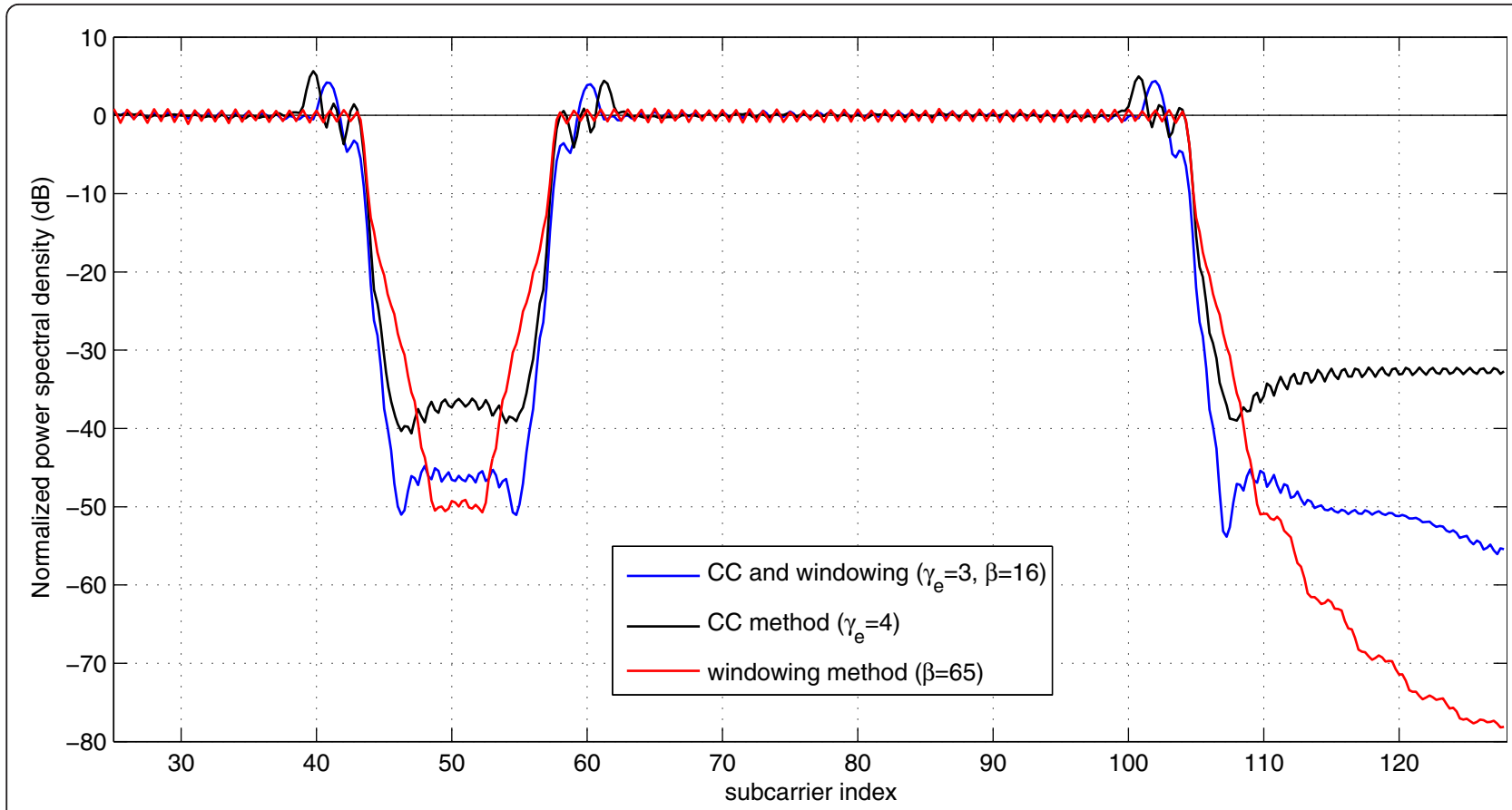

Figure 3 Normalized PSD of the NC-OFDM SU transmission signal in the considered experimental scenario with one narrow-band PU in case of the application of CC, W and combined CC and W methods.

attenuation, but it requires several frequency guard bands for the OOB attenuation slope. Thus, it is potentially unsuitable for protecting narrowband PU signals from unintentional secondary OB interference. Conversely, the CC method alone results in a relatively steep OOB power reduction, but the resulting OOB attenuation is not very high. The combination of both methods provides decent performance in terms of high and steep OOB attenuation, thus confirming that such a combination of these methods possesses the potential for protecting both wideband and narrowband PU signals employing strict requirements with respect to the signal-to-interference-power ratio. According to our other experiments for QAM/PSK schemes and to their results not presented here, the normalized PSD plots are very similar.

In Figure 4, several of the system performance metrics described above, such as the SOR for $p$ sor $=10^{-1}$, PAPR increase for $p_{\text {PAPR }}=10^{-3}$, SNR loss for BER $=10^{-4}$, and OOB power attenuation, are presented in relation to the optimization constraint parameter $\mu \in\left\langle 10^{-6}, 10^{\circ}\right\rangle$. Thus, the optimization procedure is considered in the range of $\mu$, defining scenarios from a weak constraint on the CCs power, close to no power-constraint, to a constraint on the strictly limited CCs power. For these performance metrics under consideration, measurements have been obtained after the transmission of $2 \times 10^{5}$ OFDM symbols. The SNR loss has been calculated at the receiver, for an example 4-paths Rayleigh-fading channel defined in Case 3 test scenario for UMTS user equipment [41]. Averaging of the results has been done using 10,000 channel realizations.

It can be observed in Figure 4 that the OOB power attenuation decreases slowly with an increase of $\mu$ for small values of $\mu$. Thus, when $\mu$ is low, there is no use in spending additional power on CCs since the spurious OOB emissions remain the same. On the other hand, low-power CCs (for high $\mu$ values) do not provide improvement in OOB power over results obtained for windowing method without the application of CCs. However, the other metrics improve when $\mu$ increases. For example, the fluctuation of SOR ranges from 13.7 to $-3.9 \mathrm{~dB}$. It is worth mentioning that the rest of the system performance metrics are calculated with respect to the reference system, which does not use windowing and $\mathrm{CCs}$ for OOB power reduction. Instead, the CCs are replaced with zeros. The significant improvement is observed in PAPR-increase value that approaches zero, when $\mu$ becomes high. Both new optimization goal defined by (11), and proposed reception algorithm have influence on the values of an SNR loss with standard detection and with our proposed detection making use of the CCs redundancy. The stronger the limit is on the CCs power (the higher $\mu$ ) the DCs power is not wasted as much on the CCs. Thus, the SNR loss changes from $4.8 \mathrm{~dB}$ for $\mu=10^{-6}$ to nearly $0 \mathrm{~dB}$ for $\mu=10^{\circ}$. 


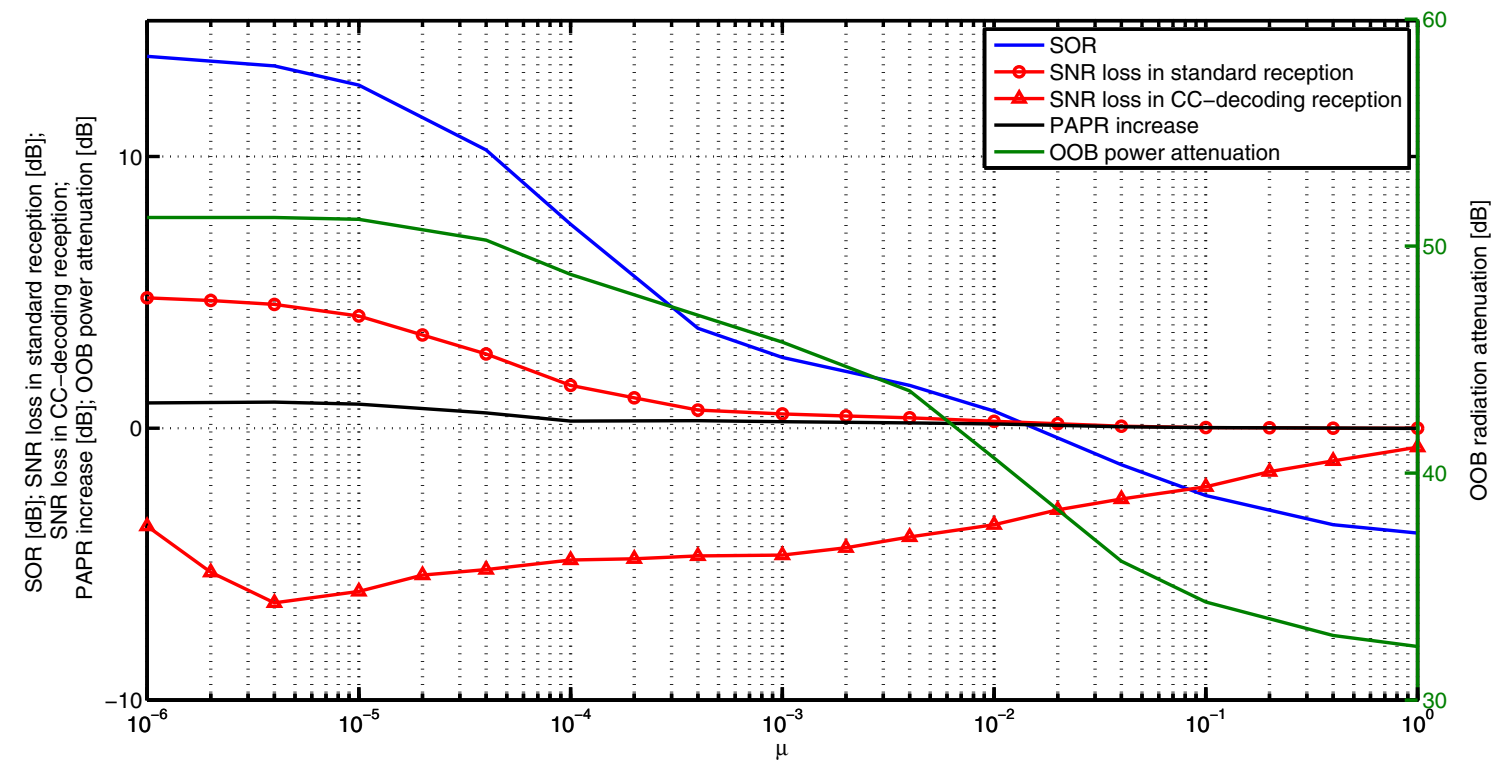

Figure 4 Simulation results for the NC-OFDM system versus the optimization factor $\mu$; SNR-loss calculated for BER $=10^{-4}$.

The results after employing our proposed detection method show that not only do the DC power levels reassigned to the CCs was recovered, but also an additional improvement was achieved thanks in part to the frequency diversity introduced by CCs treated as parity symbols of the block code. We observe that the coding gain for $\mathrm{BER}=10^{-4}$ (with respect to system without CCs) varies from $6.42 \mathrm{~dB}$ for $\mu=4 \times 10^{-6}$ to $0.7 \mathrm{~dB}$ for $\mu=1$. For very low values of $\mu\left(\mu<4 \times 10^{-6}\right)$, the SNR loss caused by the introduction of CCs becomes higher than can be compensated for even by using high power CCs, which yields a coding gain decrease. The results presented in Figure 4 show that our reception algorithm making use of the CCs redundancy yields decent performance even in the assumed case of large fragmentation of available (not occupied by the PUs) frequency bands.

\section{Real-world experimental results}

\subsection{Implementation setup}

One application for the deployment of CR systems and DSA networks is the opportunistic spectral usage of unoccupied portions of the TV frequency bands by future mobile radio systems such as long term evolution (LTE) mobile radio communication [42]. A television whitespace (TVWS) is a region of wireless frequencies where several digital video broadcasting-terrestrial (DVB-T) channels are not used by a licensed transmission, and therefore can be temporarily borrowed by TVWS devices capable of operating in these bands as long as they respect the limits concerning the maximum allowable transmit power in this area, as well as the level of their OOB interference power.

Moreover, it is envisioned that wireless microphones will be operating in these TVWS regions and associated frequency bands, as well as other wireless devices used for PMSE [43]. Although several spectrum regulators anticipate reserving one TV channel for the exclusive access by PMSE equipment (e.g., U.K. Ofcom reserves channel 38), it is anticipated to be not sufficient for large events that commonly use over 100 wireless microphones. Hence, PMSE devices may be using other channels as well. In order to address this application scenario, we consider the PU signals in this scenario to consist of a DVB-T transmission using an $8 \mathrm{MHz}$ channel and a PMSE transmission possessing a $200 \mathrm{kHz}$ bandwith. Moreover, the LTE transmissions are considered to be SU signals in these frequency bands. In particular, our tested system implements the LTE-like transmission with $N=512$ subcarriers, with the possibility of turning some of the subcarriers off, as well as using some of them as the CCs for OOB interference reduction. The subcarrier spacing is $15 \mathrm{kHz}$, and the useful spanned band is $7.68 \mathrm{MHz}$. The CP duration in samples $N_{\mathrm{CP}}=N / 16$ has been used, and the binary phase shift keying (BPSK) signalling has been applied at each subcarrier.

Note that for our SU system the PMSE band spans over 14 subcarriers, and therefore such a PMSE 
transmission is considered as narrowband PU signal. If we consider channelization of the available subcarriers in blocks of 16 subcarriers, one block of subcarriers has to be deactivated in order to protect such a narrowband PU signal when detected. Our second type of PU signal, the DVB-T system uses at minimum $8 \mathrm{MHz}$ channel, and thus more than the assumed SU bandwidth of 7.68 MHz. Therefore, it is considered to be a wideband PU signal, and if such a PU signal is detected, its channel must remain unoccupied by the SU system.

Employing the assumption that the LTE system is considered to be an SU signal, we investigate the OOB interference suppression taken from this system SEM. In particular, our goal is to achieve a $59 \mathrm{~dB}$ OOB interference power attenuation below the PSD level of data carriers for the first use-case of the high-power transmitter and call it use-case 1. Note, that the minimum required suppression defined in the LTE Base Station (BS) SEM employs this value, i.e., $59 \mathrm{~dB}$. In our second use-case, called use-case 2, $26 \mathrm{~dB}$ will be the required $\mathrm{OOB}$ power attenuation, which is the typical value for the LTE user equipment transmitter that has to be obeyed in adjacent channels. Note that in order to protect various types of PU signals present in the spectrum, their required signal-to-interference ratio must be considered together with the signal attenuation between the SU transmitter and the PU receiver. Moreover, the PU receive filters parameters and their sensitivity have to be taken into account.

It is envisioned that the TVWS geolocation databases will provide the information on the maximum in-band and $\mathrm{OOB}$ power allowable at the specific location for the specific devices and services. Here, we assume that the PU signals and SU signals are located at a distance that allows the use of the standard SEMs for the reduction of the OOB interference. Nevertheless, our proposed shaping mechanism is designed so that it can fit flexibly to any existing SEM requirement and the OOB power suppression requirements can be changed dynamically when a new PU is detected in the adjacent channel or in the middle of transmission band of the SU.

In order to evaluate the flexibility of our spectrum shaping algorithms, we consider the following four scenarios of the PU and SU coexistence, namely:

- Scenario 1: The SU system occupies continuous bandwidth, with only DC carrier turned off. The DVB-T systems or densely located PMSE devices (the PUs) are detected to operate on both sides of the SU's band, which uses subcarriers of indices: $\{-100, \ldots,-1\} \cup\{1, \ldots, 50\}$. The OOB power reduction mechanisms have to be used on both sides of the SU band.
- Scenario 2: Outer wide-band PUs (DVB-T) are detected and one narrow-band PU (PMSE device) in the middle of the SU transmission band (16 subcarriers turned off). The indices of the SU's used subcarriers are: $\{-100, \ldots,-8\} \cup\{9, \ldots, 50\}$.

- Scenario 3: Outer wide-band PUs (DVB-T) are detected and two narrow-band PU using and noncontiguous bands (PMSE devices) inside the SU's band. The indices of the SU's used subcarriers are: $\{-$ $100, \ldots,-8\} \cup\{9, \ldots, 50\} \cup\{67, \ldots, 100\}$.

In each scenario, the same number of CCs are committed to each edge of the data subcarriers blocks, e.g., two CCs are used at each edge of the data block in scenario 2, thus the indices of the CCs are: $\{-100,-99,-9$, $-8,9,10,49,50\}$. For the purpose of the spectrum windowing, the Hanning window has been chosen due to its relatively high OOB interference attenuation. The parameters of our experimental OFDM-based secondary wireless access system implemented for this project are given in Table 2. The potential rate-loss values due to the usage of the CCs (defined by (16)) are also presented.

\subsection{Experiment outcomes}

The spectrally agile OFDM experimental testbed developed at Poznan University of Technology utilizes the IRIS SDR platform [44]. IRIS was developed at Trinity College Dublin, and is a GPP-based rapid prototyping and deployment system. The building blocks of the radio components in a transceiver chain are written in $\mathrm{C}++$. Extensible Markup Language (XML) is used to specify the signal chain construction and characteristics. The usability of this platform for demonstration of the OFDM signal spectrum shaping based on filtering has been described in [45]. Using this testbed, the IRIS SDR platform has been used in conjunction with the RF hardware front-end USRP N210 and its daughterboard XCVR2450. The transmitted signal spectrum at the output of the USRP front-end has been measured using a Rhode \& Schwarz spectrum analyzer. Additionally, the transmit signal PSD at the output of the IRIS SW platform (at the input of the USRP) has been analyzed in MATLAB. The implementation setup of this testbed is shown in Figure 5.

In order to allow for the online reconfiguration, computationally efficient fast algorithm for optimization described in Section 4 has been applied, whose solution is given by formula (12). The most computationally complex operation, the matrix pseudoinverse, has been performed using CLAPACK [46] library. Other operations, i.e., matrix-matrix or matrix-vector multiplication, have been performed using self-built functions. However, their performance can be improved using low level 
Table 2 The parameters of the proposed OOB power suppression method (CC and windowing) for the considered experimental scenarios and use-cases (use-case 1 and use-case 2: 59 and $26 \mathrm{~dB}$ of the OOB power suppression, respectively)

\begin{tabular}{|c|c|c|c|c|c|c|c|c|c|}
\hline \multirow[t]{2}{*}{ Scenarios } & \multirow[t]{2}{*}{ Indices of used subcarriers } & \multicolumn{4}{|c|}{ Use-case 1} & \multicolumn{4}{|c|}{ Use-case 2} \\
\hline & & $\beta$ & $\gamma_{\mathrm{e}}$ & $\mu$ & $R_{\text {loss }}[\%]$ & $\beta$ & $\gamma_{\mathrm{e}}$ & $\mu$ & $R_{\text {loss }}[\%]$ \\
\hline Scenario 1 & $\{-100, \ldots,-1\} \cup\{1, \ldots, 50\}$ & 64 & 6 & 0.001 & 17.95 & 4 & 2 & 0.07 & 3.38 \\
\hline Scenario 2 & $\{-100, \ldots,-8\} \cup\{9, \ldots, 50\}$ & 96 & 4 & 0.001 & 25 & 4 & 2 & 0.09 & 6.613 \\
\hline Scenario 3 & $\{-100, \ldots,-8\} \cup\{9, \ldots, 50\} \cup\{67, \ldots, 100\}$ & 96 & 4 & 0.001 & 27.07 & 4 & 2 & 0.09 & 7.78 \\
\hline
\end{tabular}

$\gamma_{\mathrm{e}}$, the number of CCs per band-edge; $\beta$, the duration of the window extension in samples; $\mu$, the factor balancing OOB radiation power suppression and CCs power, $R_{\text {loss, }}$ potential rate loss

specialized libraries such as BLAS. Our SU transmitter has been constructed to be fully reconfigurable, i.e., the indices of used data carriers, the numbers of CCs at each subcarriers block edge $\gamma_{\mathrm{e}}$, or the window duration can be changed through the XML file.

The USRP interpolates the transmit signal in the field programmable gate array (FPGA) unit, but the interpolation filters are not very flat in their passband, especially when higher sampling rates are used, and therefore, some stages of the interpolation filters have to be turned off. To present reliable results, this threat had to be avoided, and thus the sampling rate was limited to
5 MSps. This changes the subcarriers spacing to about $9.8 \mathrm{kHz}$ while other parameters of OFDM modulation, e.g., CP length or number of subcarriers, are kept the same. Moreover, the PU bandwidth was also proportionally downscaled. Thus, the subcarriers block that needs to be turned off to protect the PMSE device (our narrow-band PU) still consisted of 16 subcarriers. The parameters of our CR transmitter implemented on the IRIS platform and of the Rhode \& Schwarz spectrum analyzer used in the experiments are shown in Table 3.

In Figures 6, 7, 8, 9, 10 and 11, we present the results of our OOB power reduction scheme based on the

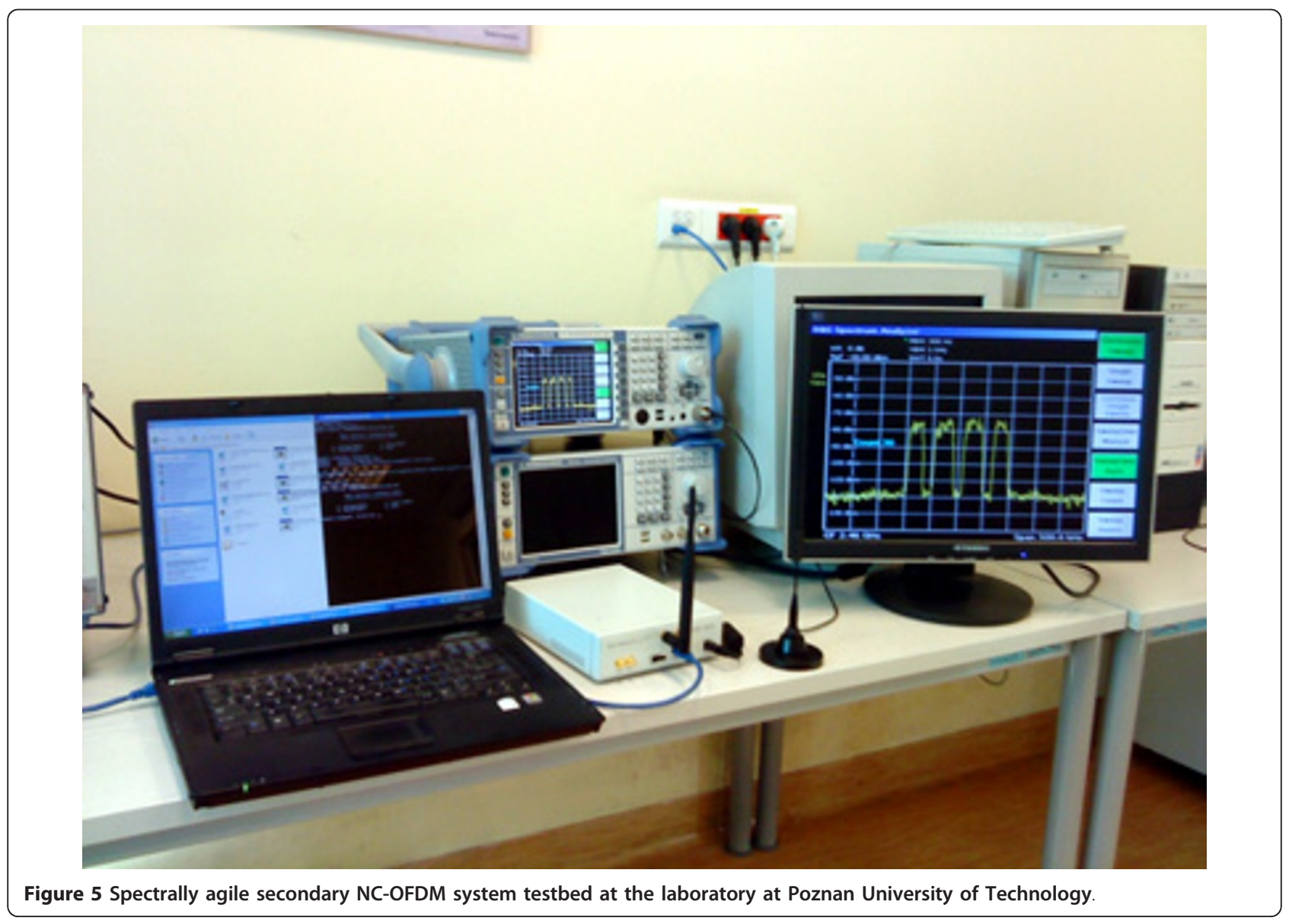


Table 3 Parameters of the experimental CR transmitter and the spectrum analyzer

\begin{tabular}{ll}
\hline Transmitter parameters & \\
The number of possible OFDM subcarriers N & 512 \\
Cyclic prefix duration in samples & N/16 \\
Maximum bandwidth & $5 \mathrm{MHz}$ \\
RF carrier frequency & $2.46 \mathrm{GHz}$ \\
Spectrum analyzer parameters & \\
SPAN & $5 \mathrm{MHz}$ \\
RBW & $10 \mathrm{kHz}$ \\
VBW & $30 \mathrm{kHz}$ \\
Number of averaging sweeps & 100 \\
\hline
\end{tabular}

modified CCs method combined with Windowing and applying new algorithms combating the spectrum overshooting problem and reducing computational complexity. Note that all these modifications and improvements necessary for practical implementation on a CR platform have been described in Section 4. Figures 6, 7, 8, 9, 10 and 11 show the PSDs of the OFDM (or NC-OFDM) $\mathrm{SU}$ transmissions under evaluation in our CR system with the wideband (DVB-T) and narrowband (PMSE) $\mathrm{PU}$ signals being protected from the interference of the high-power (BS) and low-power (user-equipment) SU transmitters. These figures show the results obtained from the scenarios under consideration (assuming the existence of PU signals) and use-cases (SEMs assumed), described in the previous subsection. The red curves present the PSD of the NC-OFDM signals with deactivated subcarriers, i.e., without any spectrum shaping method. In each plot, one can see that the sidelobe levels of the SU transmit signal without OOB power reduction possess relatively high power levels, i.e., only $20 \mathrm{~dB}$ below the power level in useful transmission band. The blue lines show the PSD of the SU signal generated at the output of the SW platform (the input to the USRP front-end) with our OOB reduction algorithm based on modified CC method with reduced complexity and windowing. There, one can see from all our evaluation scenarios that the required $\mathrm{OOB}$ power levels (either $59 \mathrm{~dB}$ or $26 \mathrm{~dB}$ below the power level in the nominal band) is achieved using adjustable parameters (the values of which are presented in Table 2). The same signal PSD at the output of the USRP front-end taken from the spectrum analyzer is plotted in black. The green line relates to the noise PSD observed in the spectrum analyzer. Note, that the USRP front-end introduces several nonlinearities that restrict the effectiveness of the OOB reduction to approximately $-45 \mathrm{~dB}$. This is the result of intermodulation products in the amplifiers and mixers of the USRP. ${ }^{a}$ This has an impact on our use-case 1 , when high $\mathrm{OOB}$ attenuation is required. For use-case 2 , when an OOB power attenuation lower than $45 \mathrm{~dB}$ is required, the influence of the intermodulation products is not crucial. Neither calibration (decrease) of

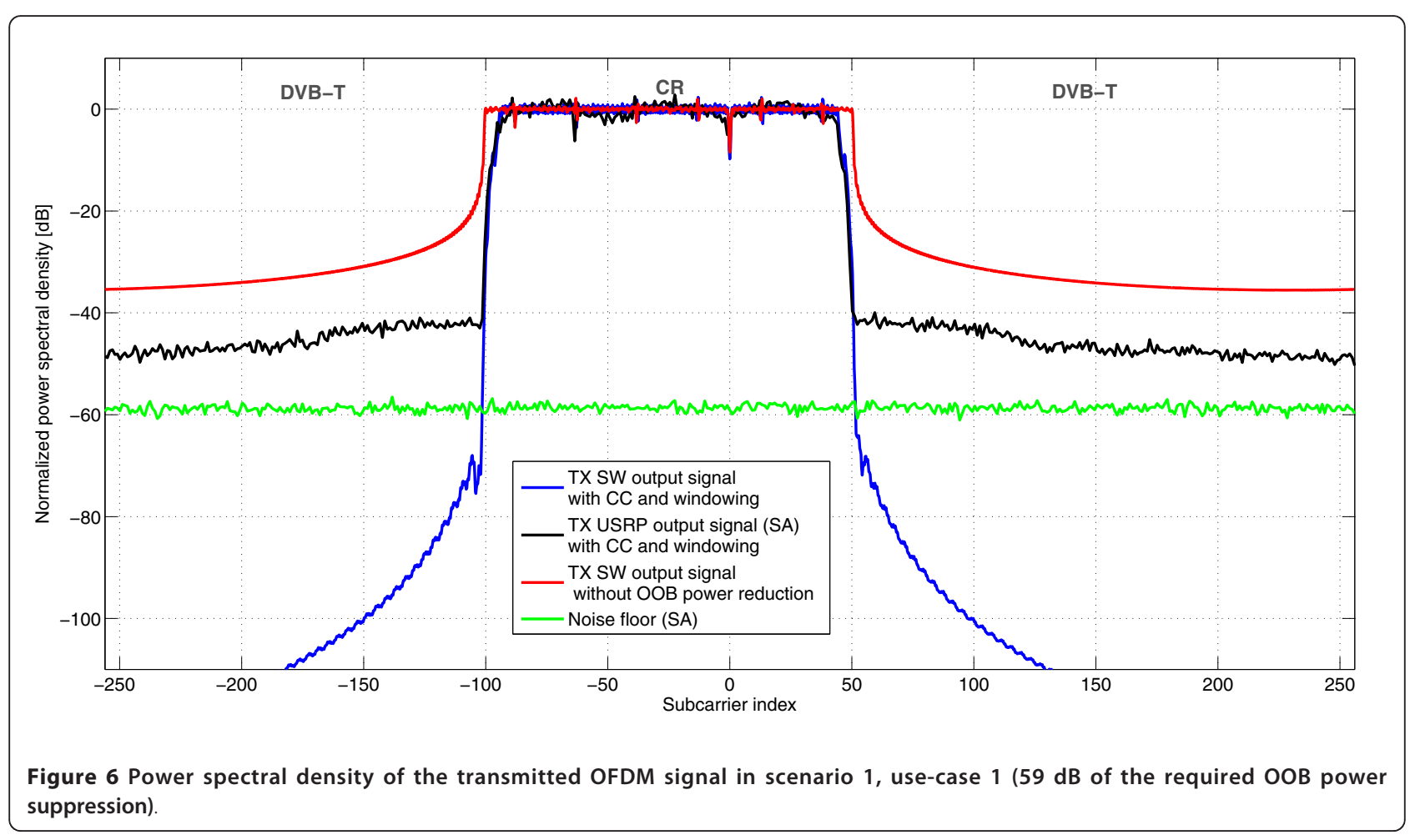




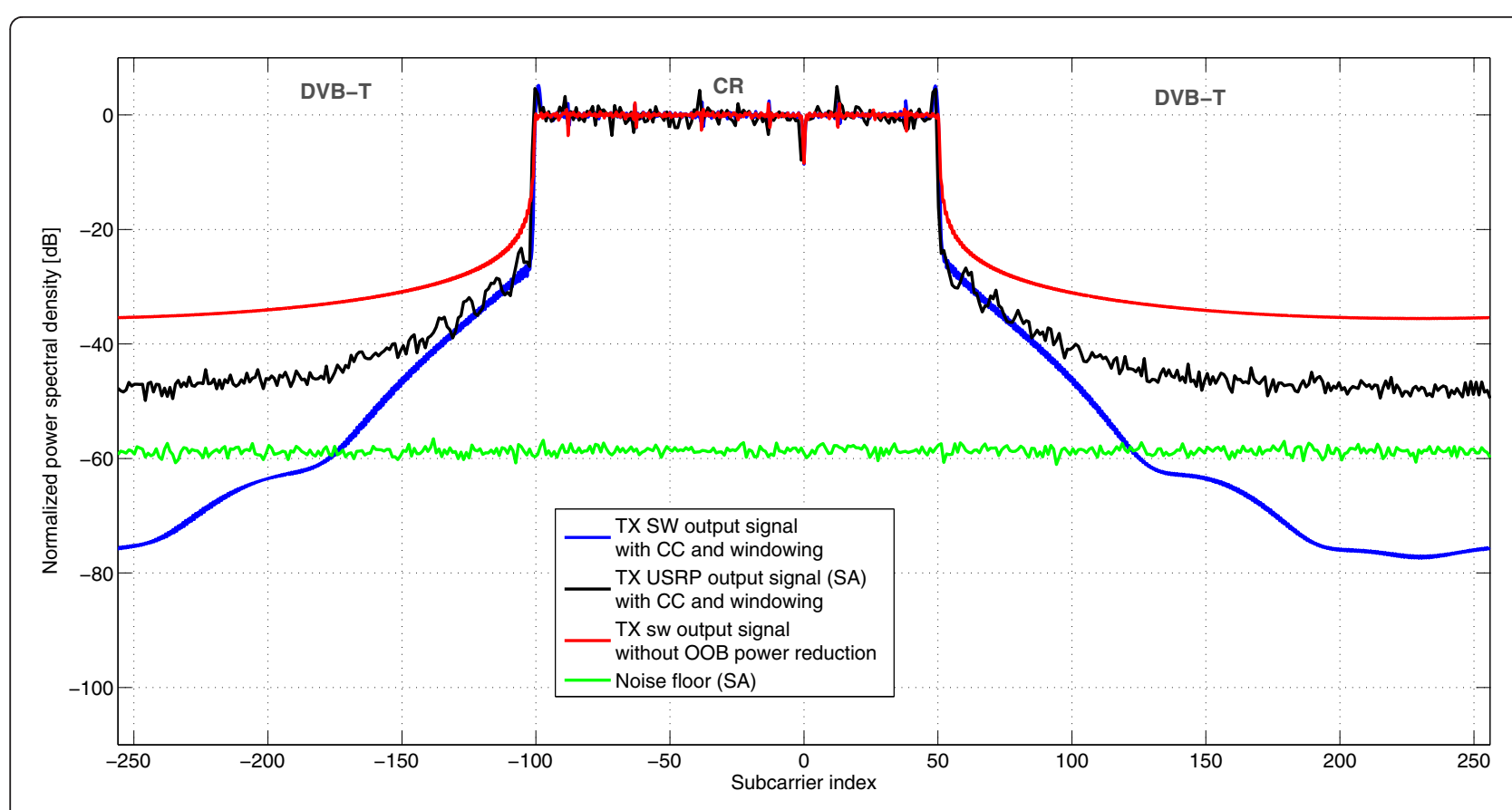

Figure 7 Power spectral density of the transmitted OFDM signal in scenario 1, use-case 2 (26 dB of the required OOB power suppression)

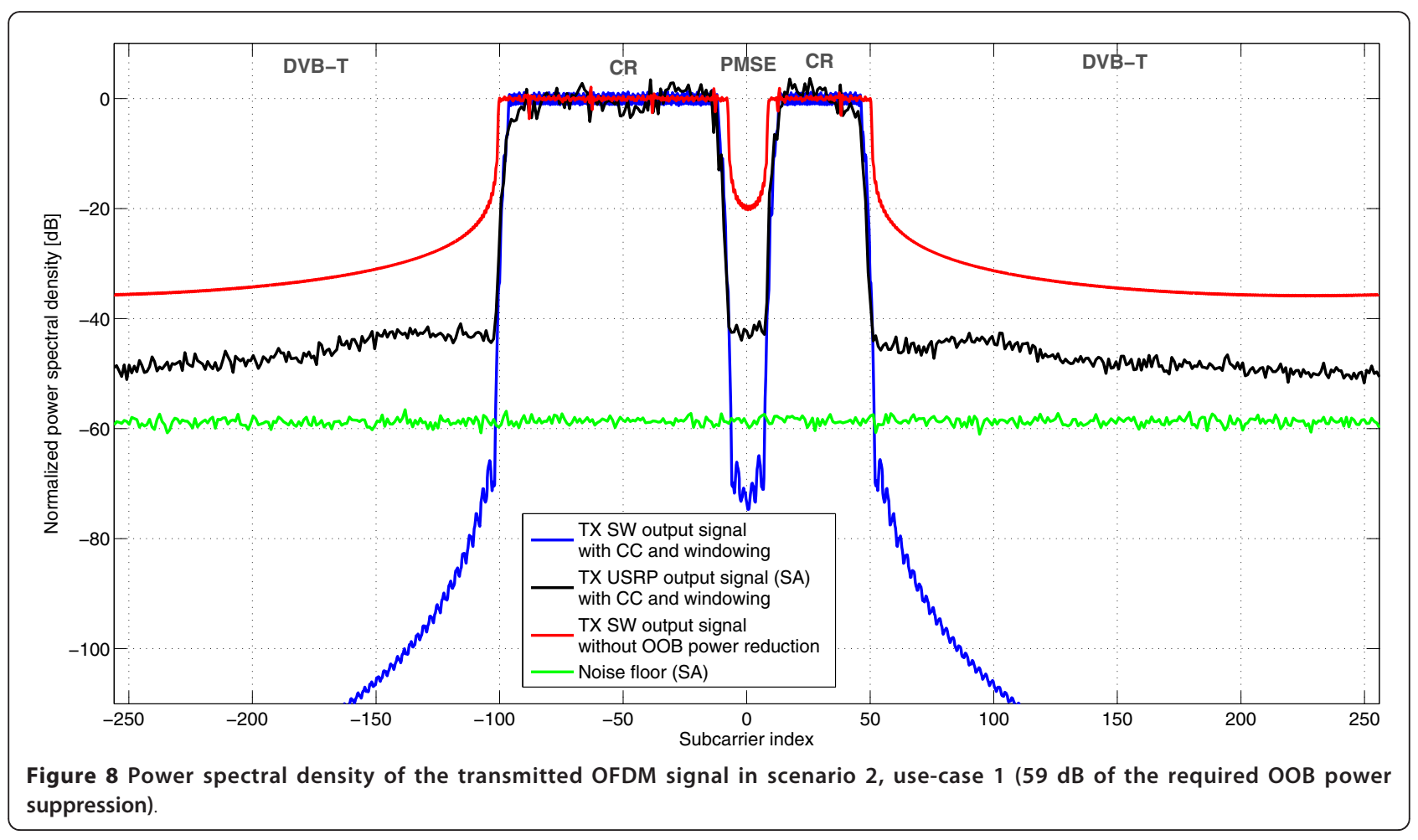



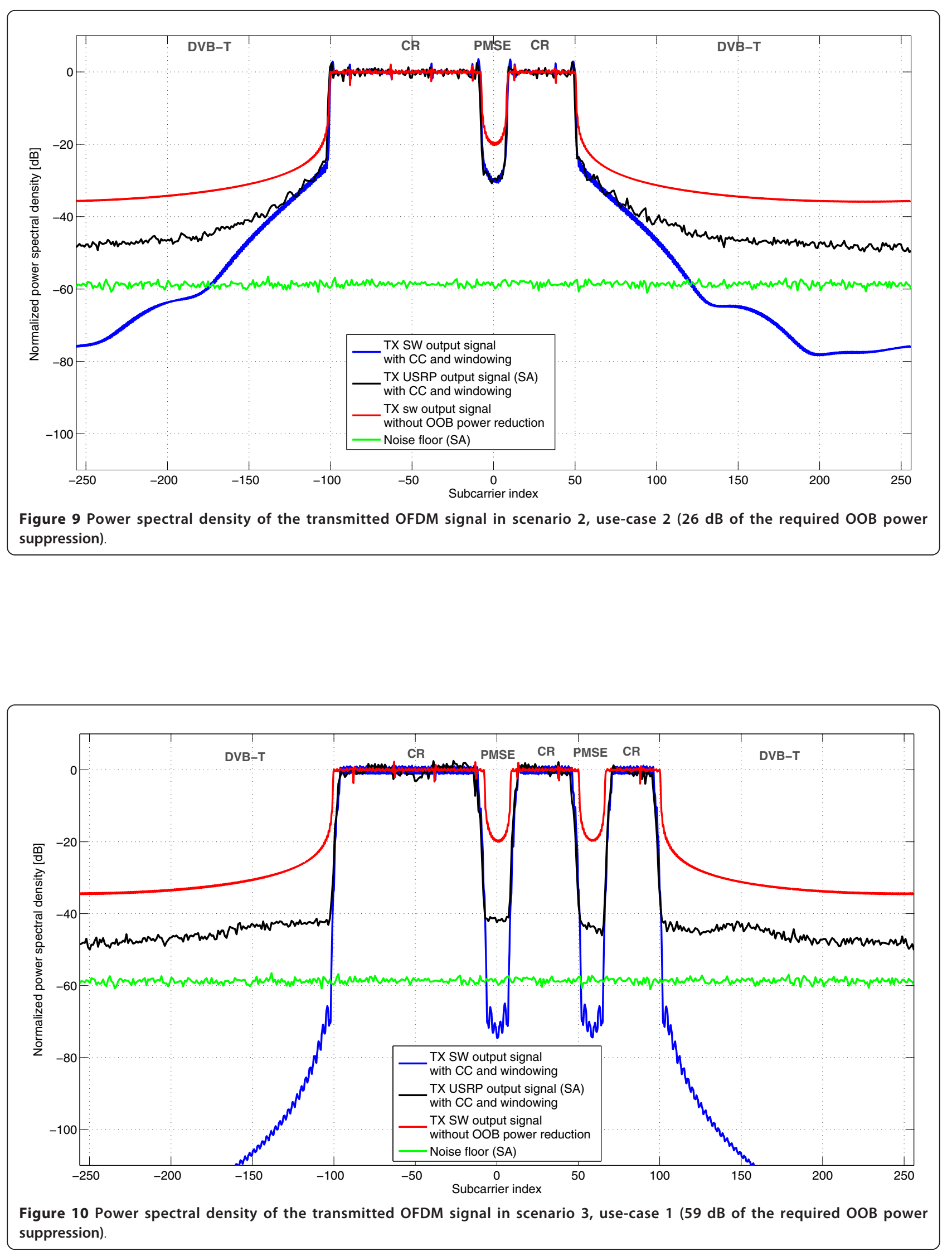


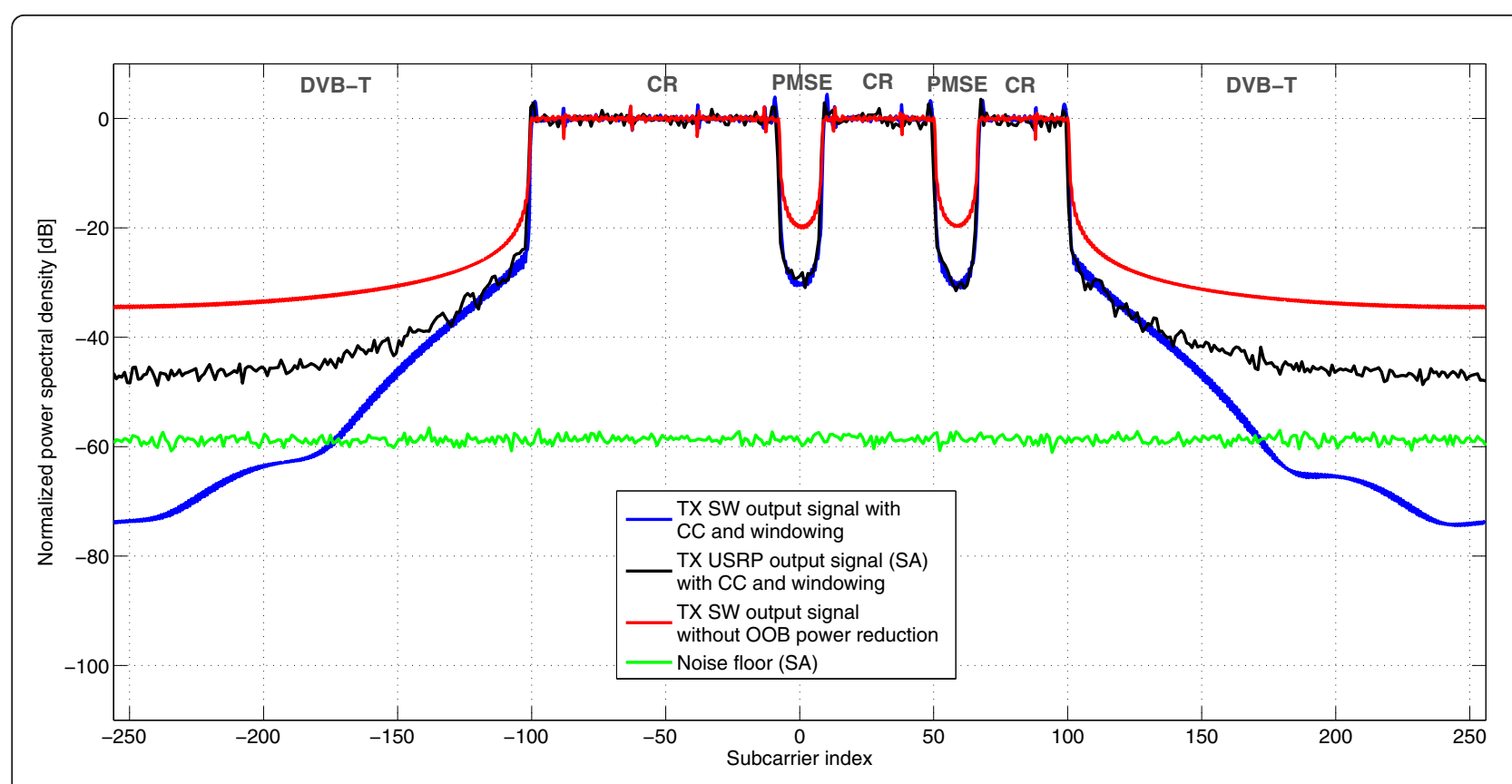

Figure 11 Power spectral density of the transmitted OFDM signal in scenario 3, use-case 2 (26 dB of the required OOB power suppression)

the transmit power nor the PAPR reduction has resulted in any positive effect to reduce these nonlinear distortions in this experimental testbed. This is due to the fact that the amplifier does not operate in its saturation region with respect to its input-output characteristic, but it is still highly nonlinear. However, the quality of the USRP platform is not at the same caliber as an actual commercial base station, where higher-quality equipment is installed, and where there are high requirements for the OOB power attenuation.

\subsection{Complexity versus interference suppression performance}

The complexity of the proposed combination of CCs and a windowing algorithm is evaluated in this subsection. At the transmitter side, the complexity is significantly reduced in comparison with the standard implementation of the CC method. It is achieved via pre-computation of the $\mathbf{W}^{(\gamma \times \alpha)}$ matrix, which has the indirect constraint on the computational complexity embedded. Thus, the optimization procedure (which is particulary complex) does not have to be performed online for every OFDM-symbol. Instead it is implemented by multiplication of the data vector by the pre-computed matrix, as described in Section 4. The most complex operation is the computation of the pseudoinverse of a $\delta+\gamma \times \gamma$ complex matrix. To achieve required accuracy of this task it is done by means of singular value decomposition whose complexity is $26 \gamma^{3}+8 \delta \gamma^{2}+4 \delta^{2} \gamma$ [47]. As $\gamma$ is a number of CCs, which is usually much lower than the number of sampling points 8 in the optimization region and lower than the number of DCs $\alpha$, this operation is feasible for the implementation even for portable devices.

The most important in our implementation was to reduce the number of computations needed for each OFDM symbol. This aim was achieved as calculation of CCs requires only $\alpha \gamma$ complex multiplications and ( $\alpha-$ 1) $\gamma$ additions. The second part of shaping is achieved by windowing that requires extending each OFDM symbol. On each side of this symbol $\beta$ samples are multiplied by windowing slopes, and thus $2 \beta$ complex-by-real multiplications are required. As subsequent OFDM symbols overlap by $\beta$ samples, $\beta$ pairs of complex numbers have to be added.

The proposed receiver discussed in Section 4 introduces some additional complexity over the standard OFDM reception. This proposed reception algorithm results in noticeable performance improvement compensating for the power-loss (power wastage) related to the use of CCs. Nevertheless it is optional, and plausible to be used by more powerful devices where the highest quality is required even at the cost of reasonably increased computational complexity. In such a case, the matrix-by-vector multiplication using $\alpha(\alpha+\gamma-1)$ additions and $(\alpha+\gamma) \alpha$ multiplications is required for each 
Table 4 Computational complexity (the number of complex additions and multiplications) of the proposed spectrum shaping algorithm with reduced complexity (per an OFDM symbol)

\begin{tabular}{|c|c|c|c|c|c|}
\hline \multirow[t]{2}{*}{ Operations } & \multicolumn{3}{|c|}{ The number of at the transmitter related to } & \multicolumn{2}{|c|}{$\begin{array}{l}\text { The number of operations at the receiver } \\
\text { operating in operations }\end{array}$} \\
\hline & $\begin{array}{l}\text { CC method } \\
\text { implementation }\end{array}$ & Windowing & $\begin{array}{l}\text { Overall spectrum } \\
\text { shaping }\end{array}$ & $\begin{array}{l}\text { Standard } \\
\text { configuration }\end{array}$ & $\begin{array}{l}\text { Proposed } \\
\text { configuration }\end{array}$ \\
\hline Complex additions & $(\alpha-1) \gamma$ & $\beta$ & $(\alpha-1) \gamma+\beta$ & 0 & $\alpha(\alpha+\gamma-1)$ \\
\hline $\begin{array}{l}\text { Complex } \\
\text { multiplications }\end{array}$ & $\alpha \gamma$ & $2 \beta$ & $\alpha \gamma+2 \beta$ & $\alpha$ & $\alpha(\alpha+\gamma)$ \\
\hline \multicolumn{6}{|l|}{$\begin{array}{l}\text { Total no. operations } \\
\text { for: }\end{array}$} \\
\hline Scenario 1, Use-case 1 & 3300 & 192 & 3492 & 138 & 41262 \\
\hline Scenario 2, Use-case 1 & 3792 & 288 & 4080 & 119 & 32011 \\
\hline Scenario 3, Use-case 1 & 7320 & 288 & 7608 & 153 & 54009 \\
\hline Scenario 1, Use-case 2 & 1164 & 12 & 1176 & 146 & 43654 \\
\hline Scenario 2, Use-case 2 & 2024 & 12 & 2036 & 127 & 34163 \\
\hline Scenario 3, Use-case 2 & 3756 & 12 & 3768 & 157 & 52909 \\
\hline
\end{tabular}

$\alpha$, the total number of $\mathrm{DCs} ; \gamma$, the total number of CCs; $\beta$, the window extension duration in samples.

OFDM symbol. The calculation of the reception (decoding) matrix is more complex as it requires computation of the pseudoinverse of the dimension $(\alpha+\gamma) \times \alpha$ complex matrix. Based on [47], one can evaluate the complexity of such an operation as $4(\alpha+\gamma)^{2} \alpha+22 \alpha^{3}$ complex operations. In case of a slowly varying channel, the update of this matrix can be performed in long periodic time intervals. Efficient algorithms for updating the pseudoinverse on the basis of a previously computed pseudoinverse also exist, and can be used for the improved reception of the NC-OFDM signal with CCs.

The computational complexity per an OFDM symbol is summarized in Table 4. For clear comparison a summarized number of complex additions and multiplications for each of our used test scenarios and use-cases is shown. We can observe that in all investigated scenarios and test cases the contribution of the $\mathrm{CC}$ method to the computational complexity of the OOB power reduction method at the transmitter is the highest. In particular, in scenario 3 and use-case 1, when strong OOB radiation suppression is required and many DCs are used the complexity of the method becomes high. The only way to obtain similar results in terms of the signal PSD with lower computational complexity is to balance windowing and CCs share in the proposed method, i.e. increase the window extenssion $\beta$ and decrease the number of CCs $\gamma$. Though, according to results of Section 4.1.1 this will provide significantly higher throughput loss required to maintain the same OOB power level. Note, that the parameters of the applied OOB power reduction method depends on the use-case and scenario to be addressed, which in turn depend on the type of a PU neighboring the SU transmission on the frequency axis and their used frequency bands.
The receiver complexity in our system depends strongly on the applied configuration. Our proposed decoding method decreases the BER significantly, but is approximately 300 times more computationally complex than standard detection. In addition, matrix-based reception depends strongly on number of DCs, so the worst result is observed in scenario 3 . Nevetheless our proposed decoding is optional, as explained previously, and thus, it can be used only by more powerful devices.

\section{Conclusion}

In this article, practical considerations with respect to the protection of primary user transmissions using CR systems performing DSA have been presented. In particular, we have identified NC-OFDM technique as a viable transmission technology capable of supporting spectrally agile communications within the context of DSA. We have also presented several methods for handling the OOB interference generated by NC-OFDM SU transmissions, observing that this type of interference can be reduced down to a level required to protect the transmission quality of the PU signals.

Moreover, we have identified the combination of the CCs and windowing methods to be particularly efficient in terms of the OOB interference power reduction, an in terms of its flexibility to adjust the SU spectrum to the required spectrum emission mask. Our proposed improvements and modifications have resulted in a reduction in the computational complexity of the energy consumption of the OOB interference reduction methods, regaining the power "wasted" by the CCs at the receiver by making use of the $\mathrm{CCs}$ correlation with the data carriers. The so-called spectrum overshooting problem has been also solved with our new proposed 
optimization technique. Simulation results show that when the optimization parameter $\mu$ is properly chosen, the performance metrics: PAPR, SOR, $\mathrm{SNR}_{\text {loss }}$ and OOB power attenuation reach their well-balanced values.

Finally, our proposed reduced-complexity, reducedpower $\mathrm{OOB}$ interference power reduction method was evaluated in several real-world scenarios involving PU and SU signals and in two basic use-cases of the required spectrum emission masks. We have considered both narrowband (e.g., PMSE) and wideband (e.g., DVBT) PU signals in these real-world experiments, along with an LTE-like SU transmission in TVWS with the possibility of aggregating the fragmented spectrum not used by the PU signals. The implementation has been conducted using an experimental SDR tested, showing exceptionally high degree of the frequency agility of our secondary-user NC-OFDM waveforms with the above presented novel practical design.

\section{Endnote}

${ }^{a}$ This effect is caused by the USRP, not the spectrum analyzer, since the noise floor of the spectrum analyzer is much lower on each plot.

\section{Acknowledgements}

This study was supported in part by the European Commission, Seventh Framework Programme, under the project COGEU (contract no. ICT-248560).

\section{Author details}

${ }^{1}$ Chair of Wireless Communications, Poznan University of Technology, Poznan, Poland ${ }^{2}$ Wireless Innovation Laboratory, Worcester Polytechnic Institute, Worcester, MA, USA

\section{Competing interests}

The authors declare that they have no competing interests.

Received: 20 May 2011 Accepted: 20 January 2012

Published: 20 January 2012

\section{References}

1. R Rajbanshi, AM Wyglinski, GJ Minden, An efficient implementation of NCOFDM transceivers for cognitive radios, in First International Conference on Cognitive Radio Oriented Wireless Networks and Communications (CROWNCOM), Mykonos Island, Greece, 1-5 (8-10 June 2006)

2. AM Wyglinski, Effects of bit allocation on non-contiguous multicarrier-based cognitive radio transceivers, in IEEE 64th Vehicular Technology Conference, Montréeal, Canada, 1-5 (25-28 September 2006)

3. H Bogucka, Directions and recent advances in PAPR reduction methods, in IEEE International Symposium on Signal Processing and Information Technology (ISSPIT), Vancouver, Canada, 821-827 (27-30 August 2006)

4. M-G Di Benedetto, P Mandarini, An application of MMSE predistortion to OFDM systems. IEEE Trans Commun. 44(11), 1417-1420 (1996)

5. Federal Communications Commission, Spectrum Policy Task Force Report, ET Docket No. 02-135, (2002)

6. Federal Communications Commission, Unlicensed Operation in the TV Broadcast Bands, ET Docket No. 04-186, (2004)

7. T Weiss, J Hillenbrand, A Krohn, FK Jondral, Mutual interference in OFDMbased spectrum pooling systems, 2004 IEEE 59th IEEE Vehicular Technology Conference, 2004, VTC 2004-Spring, Milan, Italy, 4, 1873-1877 (17-19 May 2004)
8. H Bogucka, AM Wyglinski, S Pagadarai, A Kliks, Spectrally agile multicarrier waveforms for opportunistic wireless access. IEEE Commun Mag. 49(6), 108-115 (2011)

9. S Pagadarai, A Kliks, H Bogucka, AM Wyglinski, Non-contiguous multicarrier waveforms in practical opportunistic wireless systems. IET Radar Sonar Navigat J. 5(6), 674-680 (2011)

10. HA Mahmoud, T Yucek, H Arslan, OFDM for cognitive radio: merits and challenges. IEEE Wirel Commun. 16(2), 6-14 (2009)

11. HA Mahmoud, H Arslan, Sidelobe suppression in OFDM-based spectrum sharing systems using adaptive symbol transition. IEEE Commun Lett. 12(2), 133-135 (2008)

12. S Pagadarai, R Rajbanshi, AM Wyglinski, GJ Minden, Sidelobe suppression for OFDM-based cognitive radios using constellation expansion, in IEEE Wireless Communications and Networking Conference, Las Vegas, USA, 888-893 (31 March-3 April 2008)

13. I Cosovic, S Brandes, M Schnell, Subcarrier weighting: a method for sidelobe suppression in OFDM systems. IEEE Commun Lett. 10(6), 444-446 (2006)

14. I Cosovic, S Brandes, M Schnell, A technique for sidelobe suppression in OFDM systems, in IEEE Global Telecommunications Conference, St. Louis, USA, 1-5 (28 November- 2 December 2005)

15. X Fu, J Wang, SQ Li, Sidelobe suppression for OFDM based cognitive radio systems, in Fourth International Conference on Communications and Networking, ChinaCOM 2009, Xi'an, China, 1-5 (26-28 August 2009)

16. I Cosovic, T Mazzoni, Suppression of sidelobes in OFDM systems by multiple-choice sequences. Eur Trans Telecommun. 17(6), 623-630 (2006)

17. D Li, X Dai, H Zhang, Sidelobe suppression in NC-OFDM systems using phase shift, in 4th International Conference on Wireless Communications, Networking and Mobile Computing, WiCOM'08, Dalian, China, 1-4 (12-14 October 2008)

18. S Ahmed, RU Rehman, H Hwang, New techniques to reduce sidelobes in OFDM system, in Third International Conference on Convergence and Hybrid Information Technology, ICCIT'08, Venice, Italy, 2, 117-121 (11-13 November 2008)

19. K Panta, J Armstrong, Spectral analysis of OFDM signals and its improvement by polynomial cancellation coding. IEEE Trans Consum Electron. 49(4), 939-943 (2003)

20. S Noreen, NZ Azeemi, A technique for Out-of-Band radiation reduction in OFDM-based cognitive radio, in IEEE 17th International Conference on Telecommunications, ICT 2010, Doha, Qatar, 853-856 (4-7 April 2010)

21. R Xu, M Chen, A precoding scheme for DFT-based OFDM to suppress sidelobes. IEEE Commun Lett. 13(10), 776-778 (2009)

22. CD Chung, Spectrally precoded OFDM with cyclic prefix, in IEEE International Conference on Communications, ICC'O7, Glasgow, Scotland, 5428-5432 (24-28 June 2007)

23. $\mathrm{D}$ Qu, Z Wang, T Jiang, Extended active interference cancellation for sidelobe suppression in cognitive radio OFDM systems with cyclic prefix IEEE Trans Veh Technol. 59(4), 1689-1695 (2010)

24. Z Wang, D Qu, T Jiang, Y He, Spectral sculpting for OFDM based opportunistic spectrum access by extended active interference cancellation, in IEEE Global Telecommunications Conference, GLOBECOM 2008, New Orleans, USA, 1-05 (30 November 2008-4 December 2008)

25. D Qu, Z Wang, T Jiang, M Daneshmand, Sidelobe suppression using extended active interference cancellation with self-interference constraint for cognitive OFDM system, in Fourth International Conference on Communications and Networking in China, China-COM 2009, Xi'an, China, 1-5 (26-28 August 2009)

26. MM Naghsh, MJ Omidi, Reduction of out of band radiation using carrier-bycarrier partial response signalling in orthogonal frequency division multiplexing. IET Commun. 4(12), 1433-1442 (2010)

27. J Van de Beek, F Berggren, N-continuous OFDM. IEEE Commun Lett. 13(1), 1-3 (2009)

28. G Bansal, MJ Hossain, VK Bhargava, Optimal and suboptimal power allocation schemes for OFDM-based cognitive radio systems. IEEE Trans Wirel Commun. 7(11), 4710-4718 (2008)

29. Q Lu, W Wang, T Peng, W Wang, Efficient multiuser water-filling algorithm under interference temperature constraints in OFDMA-based cognitive radio networks, in International Symposium on Microwave, Antenna, Propagation and EMC Technologies for Wireless Communications, Hangzhou, China, 174-177 (16-17 August 2007) 
30. Z Yuan, AM Wyglinski, On sidelobe suppression for multicarrier-based cognitive radio transceivers. IEEE Trans Veh Technol. 59(4), 1998-2006 (2010)

31. MS El-Saadany, AF Shalash, M Abdallah, Revisiting active cancellation carriers for shaping the spectrum of OFDM-based cognitive radios, in IEEE Sarnoff Symposium, Princeton, USA, 1-5 (30 March 2009-1 April 2009)

32. S Brandes, I Cosovic, M Schnell, Reduction of out-of-band radiation in OFDM systems by insertion of cancellation carriers. IEEE Commun Lett. 10(6), 420-422 (2006)

33. H Yamaguchi, Active interference cancellation technique for MB-OFDM cognitive radio, in 34th European Microwave Conference, Amsterdam, Netherlands, 1105-1108 (13 October 2004)

34. HA Mahmoud, H Arslan, Spectrum shaping of OFDM-based cognitive radio signals, in IEEE Radio Wireless Symposium, Orlando, USA, 113-116 (January 2008)

35. S Brandes, I Cosovic, M Schnell, Reduction of out-of-band radiation in OFDM based overlay systems, in DySPAN 2005, Baltimore, USA, 662-665 (8-11 November 2005)

36. N Sokhandan, SM Safavi, Sidelobe suppression in OFDM-based cognitive radio systems, in 10th International Conference on Information Sciences Signal Processing and their Applications (ISSPA), Kuala Lumpur, Malaysia, 413-417 (10-13 May 2010)

37. SG Huang, CH Hwang, Improvement of active interference cancellation: avoidance technique for OFDM cognitive radio. IEEE Trans Wirel Commun. 8(12), 5928-5937 (2009)

38. Z Wang, GB Giannakis, Complex-field coding for OFDM over fading wireless channels. IEEE Trans Inf Theory 49(3), 707-720 (2003)

39. IEEE Standard for Information Technology-Telecommunications and information exchange between systems-Local and metropolitan area networks-Specific requirements- Part 11: wireless LAN Medium Access Control (MAC) and Physical Layer (PHY) Specifications IEEE, IEEE Std 802.112007 (Revision of IEEE Std 802.11-1999)

40. 3GPP, TS 36.101, Evolved Universal Terrestrial Radio Access (E-UTRA); User Equipment (UE) radio transmission and reception (Release 9)

41. 3GPP TS 25.101 v. 8.4.0 Release 8; UMTS; User Equipment radio transmission and reception (FDD) (October 2008)

42. M Fitch, M Nekovee, S Kawade, K Briggs, R MacKenzie, Wireless service provision in TV white space with cognitive radio technology: A telecom operator's perspective and experience. IEEE Commun Mag. 49(3), 64-73 (2011)

43. T Erpek, MA Mchenry, A Stirling, Dynamic spectrum access operationa parameters with wireless microphones. IEEE Commun Mag. 49(3), 38-45 (2011)

44. PD Sutton, J Lotze, H Lahlou, SA Fahmy, KE Nolan, B Ozgul, TW Rondeau, J Noguera, LE Doyle, Iris: an architecture for cognitive radio networking testbeds. IEEE Commun Mag. 48(9), 114-122 (2010)

45. P Sutton, B Ozgul, I Macaluso, L Doyle, OFDM pulse-shaped waveforms for dynamic spectrum access networks, in DySPAN 2010, Singapore, 1-2 (6-9 April 2010)

46. E Anderson, Z Bai, C Bischof, S Blackford, J Demmel, J Dongarra, J Du Croz, A Greenbaum, S Hammarling, A McKenney, D Sorensen, LAPACK Users' Guide (Society for Industrial and Applied Mathematics, Philadelphia, 1999)

47. GH Golub, CF Van Loan, Matrix Computations (The Johns Hopkins University Press, Baltimore, 1996)

doi:10.1186/1687-1499-2012-23

Cite this article as: Kryszkiewicz et al.: Protection of primary users in dynamically varying radio environment: practical solutions and challenges. EURASIP Journal on Wireless Communications

and Networking 2012 2012:23.

\section{Submit your manuscript to a SpringerOpen ${ }^{\circ}$ journal and benefit from:}

- Convenient online submission

- Rigorous peer review

- Immediate publication on acceptance

- Open access: articles freely available online

- High visibility within the field

- Retaining the copyright to your article

Submit your next manuscript at $\gg$ springeropen.com 